\title{
Von Neumann-Gale Model, Market Frictions, and Capital Growth
}

DOI:

10.1080/17442508.2020.1718152

\section{Document Version}

Accepted author manuscript

Link to publication record in Manchester Research Explorer

\section{Citation for published version (APA):}

Babaei, E., Evstigneev, I. V., Schenk-hoppé, K. R., \& Zhitlukhin, M. (2020). Von Neumann-Gale Model, Market Frictions, and Capital Growth. Stochastics. https://doi.org/10.1080/17442508.2020.1718152

\section{Published in:}

Stochastics

\section{Citing this paper}

Please note that where the full-text provided on Manchester Research Explorer is the Author Accepted Manuscript or Proof version this may differ from the final Published version. If citing, it is advised that you check and use the publisher's definitive version.

\section{General rights}

Copyright and moral rights for the publications made accessible in the Research Explorer are retained by the authors and/or other copyright owners and it is a condition of accessing publications that users recognise and abide by the legal requirements associated with these rights.

\section{Takedown policy}

If you believe that this document breaches copyright please refer to the University of Manchester's Takedown Procedures [http://man.ac.uk/04Y6Bo] or contact uml.scholarlycommunications@manchester.ac.uk providing relevant details, so we can investigate your claim.

\section{OPEN ACCESS}




\title{
Von Neumann-Gale model, market frictions, and capital growth
}

\author{
Esmaeil Babaei ${ }^{\mathrm{a}}$, Igor V. Evstigneev ${ }^{\mathrm{a}}$, Klaus Reiner Schenk-Hoppéa ${ }^{\mathrm{a}}$, \\ Mikhail Zhitlukhin ${ }^{\mathrm{b}}$ \\ ${ }^{a}$ Economics, University of Manchester, Oxford Road, Manchester M13 9PL, UK \\ bSteklov Mathematical Institute, Russian Academy of Sciences, 8 Gubkina St., Moscow, \\ 119991, Russia
}

\author{
ARTICLE HISTORY \\ Compiled January 15, 2020
}

\begin{abstract}
The aim of this work is to extend the classical capital growth theory pertaining to frictionless financial markets to models taking into account various kinds of frictions, including transaction costs and portfolio constraints. A natural generalization of the notion of a benchmark investment strategy (Platen, Heath and others) is proposed, and it is shown how such strategies can be used for the analysis of growth-optimal investments. The analysis is based on the classical von Neumann-Gale model of economic growth, a stochastic version of which is used in this study as a framework for the modeling of financial markets with frictions.
\end{abstract}

\section{KEYWORDS}

Capital growth theory, transaction costs, random dynamical systems, convex multivalued operators, von Neumann-Gale dynamical systems, rapid paths.

\section{Introduction}

Capital growth theory deals with the following multiperiod investment problem: starting from some initial wealth available at time 0, find a self-financing trading strategy that maximizes the long-run growth rate of investor's wealth. This problem has been investigated by various authors: Kelly [43], Latané [47], Breiman [8], Thorp [74], Ziemba and Vickson [77, 78], Algoet and Cover [3], MacLean et al. [53-55], Hakansson and Ziemba [30], and others (the state of the art in the field is reviewed in MacLean et al. [56]). However, for the most part, results available in this literature pertain to frictionless markets. Some specialized models of markets with frictions have been studied, e.g., by Hausch and Ziemba [31], Taksar et al. [73], Iyengar and Cover [34], Akian et al. [1], and Iyengar [35].

To extend capital growth theory to models of asset markets with frictions, we use the mathematical framework of von Neumann-Gale dynamical systems. Such systems are described in terms of set-valued operators specifying for every state "today" a set of possible states "tomorrow". Characteristic features of the operators associated with von Neumann-Gale systems are certain properties of convexity and homogeneity. The original theory of von Neumann-Gale dynamics (von Neumann [79], Gale [26] and Rockafellar [66]) aimed basically at the mathematical modeling of economic growth. 
This theory, in its classical form, was purely deterministic: it did not reflect the influence of random factors on economic growth. The importance of taking these factors into account was realized early on. In the 1970s, Dynkin [20-22], Radner [64, 65] and their research groups made first steps in developing stochastic analogues of the von Neumann-Gale growth model. The initial attack on the problem left many questions unanswered since studies in this direction faced serious mathematical and conceptual difficulties. Substantial progress was made only in the 2000s [5, 23, 24], when new mathematical techniques were developed that made it possible to resolve a number of fundamental problems in the field.

A new stage in the theory of von Neumann-Gale systems began when Dempster et al. [17] observed that stochastic systems of the von Neumann-Gale type can serve as a natural and convenient framework for the modeling of financial markets with frictions. The first results in this direction obtained in [17] were concerned with no-arbitrage pricing and hedging in markets with proportional transaction costs. Extensions of these results to more general models, taking into account market interactions, were given by Evstigneev and Zhitlukhin [25].

The first applications of von Neumann-Gale dynamics to capital growth theory under proportional transaction costs were provided by Bahsoun et al. [6]. The main focus of that work was on the analysis of rapid paths in von Neumann-Gale systems, generating in financial market models benchmark strategies (numeraire portfolios), see Platen and Heath [62] and Long [52], and their applications in the theory of growthoptimal investments. In the model examined in [6], as in many other discrete-time capital growth models, short sales were ruled out, so that admissible portfolios were represented by non-negative vectors. Mathematically, this means that the state space of the von Neumann-Gale dynamical system under consideration is the non-negative cone $\mathbb{R}_{+}^{n}$ in the linear space $\mathbb{R}^{n}$.

In real financial markets, short sales are typically allowed but restricted by various trading rules (which might be different for different stock exchanges). The most common rule of this kind is expressed in terms of margin requirements, stating that only those portfolios are admissible for which at any moment of time the value of all long positions exceeds the value of all short positions with some excess (margin). In this work, we develop a capital growth model described in terms of a class of von Neumann-Gale dynamical systems in which short selling is allowed under some constraints including, in particular, margin requirements. We assume that the sets of admissible portfolio vectors, as well as self-financing constraints, are described by random cones depending on stochastic factors influencing the market. A crucial mathematical assumption under which our theoretical tools are applicable is the condition that the cones under consideration are polyhedral, i.e., generated by a finite number of (possibly random) extreme vectors. Under this condition, we show that the main results of $[5,6,24]$ can be extended to our more general model, and moreover, deduced from those in the papers cited. From the mathematical point of view, this assumption might seem restrictive, but it is acceptable in the applied perspective, since most, if not all, common models of financial markets with frictions satisfy this requirement.

The paper is organized as follows. Section 2 describes the von Neumann-Gale dynamical systems we deal with. In Section 3 the main assumptions and results are formulated. Sections 4 and 5 provide proofs of the main results. In Section 6 , we apply the general results obtained to a specialized model that covers most of the known examples and applications. The Appendix contains some general mathematical facts used in this work. 


\section{Von Neumann-Gale dynamical systems in the financial context}

A von Neumann-Gale multivalued dynamical system is defined by a sequence of cones ${ }^{1}$ $X_{t}, t=0,1,2, \ldots$, in linear spaces and cones

$$
G_{t} \subseteq X_{t-1} \times X_{t}, t=1,2, \ldots
$$

Elements of $X_{t}$ are states of the system at time $t, X_{t}$ are state spaces, and $G_{t}$ are transition cones. Sequences $x_{0}, x_{1}, \ldots$ such that

$$
\left(x_{t-1}, x_{t}\right) \in G_{t}, t=1,2, \ldots,
$$

are called paths (trajectories) of the dynamical system.

A stochastic von Neumann-Gale dynamical system is defined as follows. Let $(\Omega, \mathcal{F}, P)$ be a complete probability space and $\mathcal{F}_{0} \subseteq \mathcal{F}_{1} \subseteq \ldots \subseteq \mathcal{F}$ a sequence of $\sigma$-algebras containing all sets in $\mathcal{F}$ of measure zero. For each $t=0,1,2, \ldots$, let $X_{t}(\omega)$ be a random closed cone in a topological linear space $L\left(X_{t}(\omega)\right.$ is the random state space at time $t)$. Further, let $G_{t}(\omega) \subseteq X_{t-1}(\omega) \times X_{t}(\omega), t=1,2, \ldots$, be random closed cones. It is assumed that the cones $X_{t}(\omega)$ and $G_{t}(\omega)$ depend $\mathcal{F}_{t^{-}}$-measurably ${ }^{2}$ on $\omega$, which means that they are determined by events occurring prior to time $t$. Let $\mathcal{L}_{t}$ $(t=0,1, \ldots)$ be a linear space of $\mathcal{F}_{t}$-measurable vector functions $x(\omega), \omega \in \Omega$, with values in $L$. We say that a vector function $x(\omega)$ is a random state of the system and write $x \in \mathcal{X}_{t}$ if $x \in \mathcal{L}_{t}$ and $x(\omega) \in X_{t}(\omega)$ almost surely (a.s.). A sequence of random states $x_{0} \in \mathcal{X}_{0}, x_{1} \in \mathcal{X}_{1}, \ldots$ is called a path of the dynamical system under consideration if

$$
\left(x_{t-1}(\omega), x_{t}(\omega)\right) \in G_{t}(\omega) \text { (a.s.). }
$$

An stationary (autonomous) version of the stochastic von Neumann-Gale dynamical system is defined as follows. Let $T: \Omega \rightarrow \Omega$ be an automorphism of the probability space $(\Omega, \mathcal{F}, P)$, i.e., a one-to-one mapping of $\Omega$ onto itself such that $T$ and $T^{-1}$ are $\mathcal{F}$-measurable and preserve the measure $P$ :

$$
P\left(T^{-1} \Gamma\right)=P(T \Gamma)=P(\Gamma), \Gamma \in \mathcal{F} .
$$

We shall say that the stochastic von Neumann-Gale dynamical system is autonomous, or stationary, if

$$
T^{-1}\left(\mathcal{F}_{t}\right)=\mathcal{F}_{t+1}, X_{t}(T \omega)=X_{t+1}(\omega), \text { and } G_{t}(T \omega)=G_{t+1}(\omega) .
$$

The mapping $T$ is interpreted as a time shift: if $\Gamma \in \mathcal{F}_{t}$ is an event occurring by time $t$, then $T^{-1}(\Gamma) \in \mathcal{F}_{t+1}$ is an analogous event occurring one unit of time later (by time $t+1)$. Autonomous systems serve as a framework for stationary models in various applications (e.g. [79], [26], [23] and [24]).

\footnotetext{
${ }^{1} \mathrm{~A}$ set in a linear space is called a (convex) cone if it contains together with any vectors $a$ and $b$ the vector $\lambda a+\mu b$, where $\lambda$ and $\mu$ are any non-negative numbers. We will assume that all the cones under consideration contain non-zero vectors.

${ }^{2} \mathrm{~A}$ set $X(\omega) \subseteq L$ is said to depend $\mathcal{F}_{t}$-measurably on $\omega$ if the graph $\{(\omega, a): a \in A(\omega)\}$ of the multivalued mapping $\omega \mapsto \bar{A}(\omega)$ belongs to the $\sigma$-algebra $\mathcal{F}_{t} \otimes \mathcal{B}(L)$, where $\mathcal{B}(\cdot)$ is the Borel $\sigma$-algebra. A random set is a set $X(\omega) \subseteq L$ depending $\mathcal{F}$-measurably on $\omega$.
} 
In this work we consider stochastic von Neumann-Gale dynamical systems for which $L$ is an $N$-dimensional linear space $\mathbb{R}^{N}$ and $\mathcal{L}_{t}$ is the space $L_{t}^{\infty}\left(\mathbb{R}^{N}\right)=$ $L^{\infty}\left(\Omega, \mathcal{F}_{t}, P, \mathbb{R}^{N}\right)$ consisting of essentially bounded $\mathcal{F}_{t}$-measurable functions with values in $\mathbb{R}^{N}$. Those systems for which $X_{t}(\omega)=\mathbb{R}_{+}^{N}$ will be called canonical. They are relatively well examined, and our central goal will be to extend the corresponding results to the general setting in which $X_{t}(\omega)$ are cones that do not necessarily coincide with $\mathbb{R}_{+}^{N}$ and, moreover, depend on $t$ and $\omega$.

In financial applications, random states $x_{t} \in \mathcal{X}_{t}$ of a von Neumann-Gale system represent (contingent) portfolios of assets that can be chosen by an investor at date $t$. These portfolios are specified by random $\mathcal{F}_{t}$-measurable vectors of dimension $N$, where $N$ is the number of assets traded at each date. Portfolio positions can be measured either in terms of "physical units" of assets, or in terms of their values. The transition cones $G_{t}(\omega)$ define self-financing constraints: a portfolio $x$ can be transferred to a portfolio $y$ at date $t$ (under transaction costs) if and only if $(x, y) \in G_{t}(\omega)$. The cones $X_{t}(\omega)$ can specify various constraints on admissible portfolios, such as short selling constraints for some or all assets, or margin requirements (long portfolio positions must compensate with a certain excess its short positions). Paths $x_{0}, x_{1}, x_{2}, \ldots$ of the dynamical system at hand are feasible (self-financing) trading strategies, describing possible scenarios of the investor's actions at the financial market influenced by random factors. The fact that $G_{t}(\omega)$ and $X_{t}(\omega)$ are cones means that the model takes into account in the most general way proportional transaction costs.

Financial market models with proportional transaction costs were discussed already in the 1970s. Their origin goes back to the consumption-investment model suggested by Magill and Constantinides in [57] as a natural generalization of the Merton model [59]. However, Leland's paper [48] was likely to be the most important for the industry because of its easy practical implementation. The approach of Leland's paper has been extended to various situations by Kabanov and Safarian [41], Denis and Kabanov [18], Lépinette [50], and others. The size of the bid-ask spreads for realistic values of transaction costs has been discussed, e.g., by Zhao and Ziemba [75, 76] and Leland [49]. An important role in establishing the field was played by the work of Jouini and Kallal [38], Pham and Touzi [61], Kabanov [39], Koehl et al. [45, 46], Cadenillas and Pliska [10], Cadenillas [9], Chalasani and Jha [12], Akian et al. [1], Bouchard and Touzi [7], Delbaen and Kabanov [16], Kabanov et al. [40], Janeček and Shreve [32], De Valliere and Kabanov [19], Liu and Loewenstein [51], Jacka and Berkaoui [36], Jacka et al. [37], Guasoni et al. [29], Albanese and Tompaidis [2], and others. For a comprehensive review of this literature we refer to the monograph "Markets with Transaction Costs" by Kabanov and Safarian [42]. For more recent work see Lépinette et al. [28], Roux and Zastawniak [68-71], Roux [67], and Kifer [44].

The generality of the von Neumann-Gale framework makes it possible to substantially enhance and broaden the scope of the models of financial markets with proportional transaction costs. In particular, one can include into consideration various kinds of portfolio constraints, such as margin requirements, specified by random cones. One can incorporate not only standard single-currency market models but also multicurrency models (see Kabanov [39], Kabanov and Safarian [42] and references therein). For various examples and applications of the von Neumann-Gale approach to questions of asset pricing and hedging see Dempster et al. [17]. The results obtained in [17] were extended by Evstigneev and Zhitlukhin [25] in two new directions: "soft" hedging in terms of risk measures (rather then a.s. superreplication) and the analysis of a system of interacting financial markets with the structure of interactions between them described in terms of a directed graph. In the present paper (see Section 6), we 
apply the general theory to a multicurrency financial market model with proportional transaction costs in which not only currencies but also dividend-paying assets (e.g. shares of stock) are traded. In this setting, interest rates for borrowing and lending might differ from each other and might be different for different currencies. The main results are concerned with capital growth theory for such markets both in stationary and nonstationary settings.

Let $X_{t}^{*}(\omega)$ denote the dual cone of $X_{t}(\omega)$ :

$$
X_{t}^{*}(\omega)=\left\{p \in \mathbb{R}^{N}: p a \geq 0, a \in X_{t}(\omega)\right\},
$$

where $p a$ is the scalar product of the vectors $p$ and $a$ in $\mathbb{R}^{N}$. For shortness, we will use the notation $L_{t}^{1}\left(\mathbb{R}^{N}\right)$ for the space $L_{1}\left(\Omega, \mathcal{F}_{t}, P, \mathbb{R}^{N}\right)$ of integrable $\mathcal{F}_{t}$-measurable vector functions with values in $\mathbb{R}^{N}$. A dual path (dual trajectory) is a sequence of vector functions $p_{1}(\omega), p_{2}(\omega), \ldots$ such that $p_{t} \in L_{t}^{1}\left(\mathbb{R}^{N}\right)$ and for almost all $\omega$ we have:

$$
p_{t}(\omega) \in X_{t-1}^{*}(\omega), t=1,2, \ldots
$$

and

$$
\bar{p}_{t+1}(\omega) b \leq p_{t}(\omega) a \text { for all }(a, b) \in G_{t}(\omega), t=1,2, \ldots,
$$

where $\bar{p}_{t+1}(\omega):=E_{t} p_{t+1}(\omega)$ and $E_{t}(\cdot)=E\left(\cdot \mid \mathcal{F}_{t}\right)$ is the conditional expectation given $\mathcal{F}_{t}$.

Note that for a canonical system, we have $X_{t-1}^{*}(\omega)=\mathbb{R}_{+}^{N}$, so that elements $p_{t}$ of a dual path are functions belonging to the non-negative cones $L_{t}^{1,+}\left(\mathbb{R}^{N}\right)$ of the spaces $L_{t}^{1}\left(\mathbb{R}^{N}\right)$.

It follows from (2) that for any path $x_{0}, x_{1}, \ldots$, the random sequence $p_{t+1} x_{t}, t=$ $0,1, \ldots$, is a supermartingale with respect to the filtration $\mathcal{F}_{1} \subseteq \mathcal{F}_{2} \subseteq \ldots$ and the random sequence $\bar{p}_{t+1} x_{t}, t=0,1, \ldots$, is a supermartingale with respect to the filtration $\mathcal{F}_{0} \subseteq \mathcal{F}_{1} \subseteq \ldots$. This is immediate from the relations:

$$
E_{t} p_{t+1} x_{t}=\bar{p}_{t+1} x_{t} \leq p_{t} x_{t-1}(\text { a.s. }), t=1,2, \ldots,
$$

and

$$
E_{t-1} \bar{p}_{t+1} x_{t} \leq E_{t-1} p_{t} x_{t-1}=\bar{p}_{t} x_{t-1} \text { (a.s.), } t=1,2, \ldots,
$$

following from (2).

A standard argument using measurable selection (see Theorem 7.1 in the Appendix) shows that (2) holds if and only if

$$
E p_{t+1}(\omega) y_{t}(\omega) \leq E p_{t}(\omega) x_{t}(\omega)
$$

for all pairs $\left(x_{t}(\omega), y_{t}(\omega)\right)$ of functions in $L_{t}^{\infty}\left(\mathbb{R}^{N}\right) \times L_{t}^{\infty}\left(\mathbb{R}^{N}\right)$ such that

$$
\left(x_{t}(\omega), y_{t}(\omega)\right) \in G_{t}(\omega) \text { (a.s.). }
$$

In the financial context, dual paths are termed consistent price systems - see Jouini and Kallal [38], Cvitanić and Karatzas [14], Schachermayer [72], Guasoni et al. [29], Kabanov and Safarian [42] and others. The coordinates $p_{t}^{i}$ of the vectors $p_{t}$ are 
interpreted as market consistent prices of assets if portfolio positions are measured in terms of units of assets. If they are measured in monetary terms, then $p_{t}^{i}$ might be interpreted as market consistent discount factors. The idea of this notion goes back to the notion of competitive prices supporting competitive paths, maximizing profits over each time period $t-1, t$ in the theory of economic dynamics, - see, e.g., Malinvaud [58], Radner [63], Gale [27], Peleg [60], Dasgupta and Mitra [15], and Clark [13]. Consistent price systems generalize the concept of an equivalent martingale measure involved in classical no-arbitrage criteria pertaining to frictionless markets; for a rigorous formulation and proof of this assertion see [17, Theorem 9.3].

A central notion in this theory is the notion of a rapid path. Let us say that a dual path $p_{1}, p_{2}, \ldots$ supports a path $x_{0}, x_{1}, \ldots$ if

$$
p_{t+1} x_{t}=1, t=0,1, \ldots \text { (a.s.). }
$$

A trajectory is called rapid if there exists a dual trajectory supporting it.

The term "rapid" is motivated by the fact that

$$
\frac{\bar{p}_{t+1} y_{t}}{p_{t} y_{t-1}} \leq \frac{\bar{p}_{t+1} x_{t}}{p_{t} x_{t-1}}=1, t=1,2, \ldots \text { (a.s.) }
$$

for each path $y_{0}, y_{1}, \ldots$ with $p_{t} y_{t-1}>0$ (see (2) and (4)). This means that the path $x_{0}, x_{1}, \ldots$ maximizes the conditional expectation given $\mathcal{F}_{t}$ of the growth rate $p_{t+1} y_{t} / p_{t} y_{t-1}$ at each time $t$, the maximum being equal to 1 . Growth rates are measured by using the random "price systems" $p_{t}$. Another justification of the above term is related to the fact that rapid paths are asymptotically growth-optimal almost surely: they exhibit the fastest growth over an infinite time horizon with probability one (see Theorem 3.4 below).

In the context of the present model, rapid paths may be regarded as analogues of benchmark strategies (numeraire portfolios), see Platen and Heath [62] and Long [52]. As we have noticed, the price system (or the system of discount factors) $\left(p_{t}\right)$ involved in the definition of a rapid path is such that the value $p_{t+1} x_{t}$ of the portfolio $x_{t}$ is always equal to one, while for any other feasible sequence $\left(y_{t}\right)$ of contingent portfolios (self-financing trading strategy), the values $p_{t+1} y_{t}$ form a supermartingale. In models with unlimited short selling (cf. [62]), one can speak of martingales rather than supermartingales.

\section{Assumptions and the main results}

For a vector $a$, let us denote by $\mathbb{B}(a, r)$ the ball $\{b:|b-a| \leq r\}$, where $|\cdot|$ is the sum of the absolute values of the coordinates of a vector. Let us introduce the following conditions.

(G1) For all $t \geq 1, \omega \in \Omega$ and $a \in X_{t-1}(\omega)$, the set $\left\{b:(a, b) \in G_{t}(\omega)\right\}$ is non-empty.

(G2) For each $t \geq 1$ there is a constant $M_{t}$ such that the set $G_{t}(\omega)$ is contained in $\left\{(a, b):|b| \leq M_{t}|a|\right\}$ for all $\omega \in \Omega$.

(G3) For every $t \geq 1$ there exist a strictly positive constant $\alpha_{t}>0$ and a bounded vector function $\hat{z}_{t}(\omega)=\left(\hat{x}_{t-1}(\omega), \hat{y}_{t}(\omega)\right)$ such that $\hat{x}_{t-1}(\omega)$ is $\mathcal{F}_{t-1}$-measurable, $\hat{y}_{t}(\omega)$ is $\mathcal{F}_{t}$-measurable and $\mathbb{B}\left(\hat{z}_{t}(\omega), \alpha_{t}\right) \subseteq G_{t}(\omega)$ for all $\omega$.

$(\mathbf{F})$ There exist $\mathcal{F}_{t}$-measurable random vectors $f_{t, k}(\omega), k=1, \ldots, K$, such that for 
each $\omega$ we have $f_{t, k}(\omega) \neq 0$,

$$
X_{t}(\omega)=\left\{a: a=\sum_{k=1}^{K} f_{t, k}(\omega) c^{k} \text { for some } c^{k} \geq 0, k=1, \ldots, K\right\}
$$

and

$$
\theta_{t}|c| \leq\left|\sum_{k=1}^{K} f_{t, k}(\omega) c^{k}\right| \leq \Theta_{t}|c|, c=\left(c^{1}, \ldots, c^{K}\right) \in \mathbb{R}_{+}^{K},
$$

where $0<\theta_{t}<\Theta_{t}(t=0,1, \ldots)$ are constants and $K$ is a natural number.

In financial terms, condition $(\mathbf{G} 1)$ states that any feasible portfolio $a \in X_{t-1}(\omega)$ can be transformed - by selling and buying assets under transaction costs - to a feasible portfolio $b \in X_{t}(\omega)$. The possibility of this transformation is expressed by the fact that $(a, b) \in G_{t}(\omega)$. The vector $b$ can be equal to zero; in this case we speak of portfolio liquidation. Assumption (G2) means the uniform boundedness of the random setvalued operator $a \longmapsto\left\{b:(a, b) \in G_{t}(\omega)\right\}$. Suppose that the self-financing conditions are defined in terms of bid and ask prices. Then (G2) holds if these prices are uniformly bounded and uniformly bounded away from zero. Condition (G3) is an assumption of non-degeneracy of the model: the cone $G_{t}(\omega)$ is assumed to have a (uniform) interior point.

Let us discuss condition $(\mathbf{F})$. The representation (5) of the cone $X_{t}(\omega)$ means that this cone is polyhedral: it is spanned on a finite set of $\mathcal{F}_{t}$-measurable random vectors $f_{t, k}(\omega) \neq 0, k=1, \ldots, K$ (generators of $X_{t}(\omega)$ ). By virtue of $(5)$, we have

$$
X_{t}(\omega)=F_{t}(\omega) \mathbb{R}_{+}^{K},
$$

where $F_{t}(\omega): \mathbb{R}_{+}^{K} \rightarrow \mathbb{R}^{N}$ is the linear operator transforming $c=\left(c^{1}, \ldots, c^{K}\right) \in \mathbb{R}_{+}^{K}$ into $a=\sum_{k=1}^{K} f_{t, k}(\omega) c^{k} \in \mathbb{R}^{N}$. The inequalities in (6) can be written

$$
\theta_{t}|c| \leq\left|F_{t}(\omega) c\right| \leq \Theta_{t}|c|, c=\left(c_{1}, \ldots, c_{K}\right) \in \mathbb{R}_{+}^{K} .
$$

For a real number $r$ define $r_{+}:=\max \{r, 0\}$ and $r_{-}:=\max \{-r, 0\}$, so that $r=r_{+}-$ $r_{-}$. If $c=\left(c_{1}, \ldots, c_{K}\right)$, then $c_{+}$and $c_{-}$stand for the vectors with the coordinates $\left(c_{k}\right)_{+}$ and $\left(c_{k}\right)_{-}$, respectively. Note that the second inequality in (8) implies the analogous inequality holding for all $c \in \mathbb{R}^{K}$, and not only for $c \in \mathbb{R}_{+}^{K}$. Indeed, we have $F_{t} c=$ $F_{t} c_{+}-F_{t} c_{-}$, and so

$$
\left|F_{t} c\right|=\left|F_{t} c_{+}-F_{t} c_{-}\right| \leq\left|F_{t} c_{+}\right|+\left|F_{t} c_{-}\right| \leq \Theta_{t}\left(\left|c_{+}\right|+\left|c_{-}\right|\right)=\Theta_{t}|c| .
$$

We formulate an assumption on the cone $X_{t}(\omega)$ that guarantees the validity of condition (6) and has a natural financial interpretation.

(M) There exists a constant $\mu_{t}>1$ such that

$$
\mu_{t} \sum_{i=1}^{N} a_{-}^{i} \leq \sum_{i=1}^{N} a_{+}^{i} \text { for all } a=\left(a^{1}, \ldots, a^{N}\right) \in X_{t}(\omega) .
$$


Proposition 3.1. If the cone $X_{t}(\omega)$ is representable in the form (5) with some generators $f_{t, k}(\omega)$, and condition $(\boldsymbol{M})$ holds, then $X_{t}(\omega)$ is representable in the form (5) with generators $f_{t, k}(\omega)$ satisfying $(6)$, i.e., $X_{t}(\omega)$ satisfies condition $(\boldsymbol{F})$.

To explain the meaning of condition (M) suppose that positions of a portfolio $a \in X_{t}(\omega)$ are measured in terms of their values (expressed in some price system). Then the sums $\sum_{i=1}^{N} a_{+}^{i}$ and $\sum_{i=1}^{N} a_{-}^{i}$ represent the total value of the long and the short positions of $a$, respectively. Condition (9) means that for admissible portfolios, the long positions must cover the short ones with a certain margin $\mu_{t}$. Margin requirements of the type (9) are quite common in financial practice. They restrict short selling to exclude bankruptcy under sudden price jumps.

Proof of Proposition 3.1. Since $f_{t, k}(\omega) \neq 0$, we can assume without loss of generality that all the generators $f_{t, k}(\omega)$ of the cone $X_{t}(\omega)$ are normalized: $\left|f_{t, k}(\omega)\right|=1$. Then the second inequality in (6) will hold with $\Theta_{t}=1$.

To prove the first inequality, consider the non-random cone $\tilde{X}_{t}=\left\{a \in \mathbb{R}^{N}: \mu_{t}\left|a_{-}\right| \leq\right.$ $\left.\left|a_{+}\right|\right\}$, so that $X_{t}(\omega) \subseteq \tilde{X}_{t}$. Observe that since $\mu_{t}>1$ we have $\tilde{X}_{t} \cap\left(-\tilde{X}_{t}\right)=\{0\}$. Therefore, the minimum of the continuous function $v\left(c, f_{1}, \ldots, f_{K}\right):=\left|\sum_{k} c_{k} f_{k}\right|$ is strictly positive on the compact set $\left\{(c, f): c \in \mathbb{R}_{+}^{K},|c|=1, f_{k} \in \tilde{X}_{t},\left|f_{k}\right|=1, k=\right.$ $1, \ldots, K\}$. Then $\theta_{t}$ can be taken equal to this minimum.

The results of this paper are concerned with general (non-stationary) and stationary von Neumann-Gale dynamical systems. The main result pertaining to the former ones is as follows.

Theorem 3.2. Let conditions $(\boldsymbol{G} \mathbf{1})-(\boldsymbol{G} 3)$ and $(\boldsymbol{F})$ hold. Let $x_{0}$ be a function in $\mathcal{X}_{0}$ such that $\mathbb{B}\left(x_{0}(\omega), \varepsilon\right) \subseteq X_{0}(\omega)$ (a.s.), where $\varepsilon$ is a strictly positive constant. Then the following assertions are valid.

(i) For each $n \geq 1$, there exists a finite rapid path of length $n$ with the initial state $x_{0}(\omega)$.

(ii) There exists an infinite rapid path with the initial state $x_{0}(\omega)$.

An important property of infinite rapid paths, which determines their role in capital growth theory, is their a.s. asymptotic optimality. A path $x_{0}, x_{1}, \ldots$ is called asymptotically growth-optimal if for any other path $y_{0}, y_{1}, \ldots$ there exists a supermartingale $\xi_{t}$ such that

$$
\frac{\left|y_{t}\right|}{\left|x_{t}\right|} \leq \xi_{t}, t=0,1, \ldots \text { (a.s.). }
$$

The property of asymptotic growth-optimality, as defined above, has the following important implications. If $\left|y_{t}\right| /\left|x_{t}\right| \leq \xi_{t}, t=0,1, \ldots$ (a.s.), where $\xi_{t}$ is a supermartingale, the following assertions hold.

(a) For any constant $a>0$

$$
P\left(\sup _{t \geq 0} \frac{\left|y_{t}\right|}{\left|x_{t}\right|} \geq a\right) \leq \frac{E \xi_{0}}{a}
$$

and, in particular, $\sup _{t}\left(\left|y_{t}\right| /\left|x_{t}\right|\right)<\infty$ a.s., i.e. no strategy can grow asymptotically faster than $x_{0}, x_{1}, \ldots$ (a.s.). 
(b) The strategy $x_{0}, x_{1}, \ldots$ maximizes a.s. the exponential growth rate:

$$
\limsup _{t \rightarrow \infty} \frac{1}{t} \ln \frac{\left|y_{t}\right|}{\left|x_{t}\right|} \leq 0 \text { a.s. }
$$

(c) For any stopping time $\tau$

$$
E \frac{\left|y_{\tau}\right|}{\left|x_{\tau}\right|} \leq E \xi_{0} \text { and } E \ln \frac{\left|y_{\tau}\right|}{\left|x_{\tau}\right|} \leq \ln E \xi_{0}
$$

Assertion (a) follows from Doob's inequality for non-negative supermartingales: $P\left(\sup _{t} \xi_{t} \geq a\right) \leq E \xi_{0} / a$. Assertion (b) is immediate from that $\sup _{t}\left(\left|y_{t}\right| /\left|x_{t}\right|\right)<\infty$ a.s. The first part of assertion (c) holds because $E \xi_{\tau} \leq \liminf _{t \rightarrow \infty} E \xi_{\tau \wedge t} \leq E \xi_{0}$ by Fatou's lemma and Doob's stopping theorem applied to bounded stopping times $\tau \wedge t$. The second part of (c) follows from there by Jensen's inequality.

Note that the above properties (a)-(c) remain valid (but maybe with different constants in the right-hand sides of the inequalities in (a) and (c)) if $\left|x_{t}\right|$ and $\left|y_{t}\right|$ are replaced by $\phi_{t}\left(\omega, x_{t}\right)$ and $\phi_{t}\left(\omega, y_{t}\right)$ respectively with any function $\phi_{t}(\omega, b)$, possibly random and depending on $t$, which satisfies the following condition.

(L) There exist non-random constants $0<l \leq L$ such that $l|b| \leq \phi_{t}(\omega, b) \leq L|b|$ for all $t, \omega$ and $b \in X_{t}(\omega)$.

As an example of such a function, we can consider the liquidation value of a portfolio $b=\left(b^{1}, \ldots, b^{N}\right) \in X_{t}(\omega)$ :

$$
\phi_{t}(\omega, b)=\sum_{i=1}^{N}\left(1-\lambda_{t, i}^{+}(\omega)\right) b_{+}^{i}-\sum_{i=1}^{N}\left(1+\lambda_{t, i}^{-}(\omega)\right) b_{-}^{i},
$$

where $0 \leq \lambda_{t, i}^{+}(\omega)<1$ and $\lambda_{t, i}^{-}(\omega) \geq 0$ are transaction cost rates for selling and buying assets assets (for details see Section 6). Conditions under which the function (10) satisfies (L) are given in Proposition 3.3 below.

Proposition 3.3. Let all the cones $X_{t}(\omega)$ be representable in the form (5) and the margin requirement $(\boldsymbol{M})$ is satisfied with $\mu_{t}=\mu$ independent of $t$. If there exist constants $\underline{\Lambda}, \bar{\Lambda}$ such that $0<\underline{\Lambda} \leq 1-\lambda_{t, i}^{+}(\omega)$ and $1+\lambda_{t, i}^{-}(\omega) \leq \bar{\Lambda}$ for all $\omega, t, i$, and $\mu \underline{\Lambda}>\bar{\Lambda}$, then the liquidation value $\phi_{t}(\omega, b)$ defined in (10) satisfies condition $(\boldsymbol{L})$.

Proof. For every $b=\left(b^{1}, \ldots, b^{N}\right) \in X_{t}(\omega)$, we have $\phi_{t}(\omega, b) \leq\left|b_{+}\right|-\left|b_{-}\right|$, so the second inequality in condition $(\mathbf{L})$ holds with $L=1$.

Let us show the first inequality in $(\mathbf{L})$. For every $b \in X_{t}(\omega)$, we have

$$
\phi_{t}(\omega, b) \geq \underline{\Lambda}\left|b_{+}\right|-\bar{\Lambda}\left|b_{-}\right| \geq(\underline{\Lambda}-\bar{\Lambda} / \mu)\left|b_{+}\right| .
$$

Using that $\left|b_{+}\right| \geq\left|b_{-}\right|$, the above inequality yields $\phi_{t}(\omega, b) \geq l|b|$ with constant $l=$ $(\underline{\Lambda}-\bar{\Lambda} / \mu) / 2>0$.

Let us introduce the following condition:

(G4) There exist a real number $\gamma>0$ and a natural number $m$ such that for every $t \geq 0$ and every random vector $y_{t} \in \mathcal{X}_{t}$, one can find random vectors $y_{t+1} \in$ 
$\mathcal{X}_{t+1}, \ldots, y_{t+m} \in \mathcal{X}_{t+m}$ satisfying

$$
\left(y_{t}, y_{t+1}\right) \in G_{t+1}(\omega), \ldots,\left(y_{t+m-1}, y_{t+m}+y\right) \in G_{t+m}(\omega) \text { (a.s.) }
$$

for each $y \in L_{t+m}^{\infty}\left(\mathbb{R}^{N}\right)$ with $|y(\omega)| \leq \gamma\left|y_{t}(\omega)\right|$ (a.s.).

Condition (G4) and its versions are used practically in all studies on von NeumannGale dynamical systems, both deterministic and stochastic ones. Mathematically, (G4) says that from any state $y_{t} \in \mathcal{X}_{t}$ of the von Neumann-Gale system at hand one can reach in $m$ steps some state $y_{t+m} \in \mathcal{X}_{t+m}$ together with any $y^{\prime} \in \mathcal{X}_{t+m}$ for which the distance $\left|y_{t+m}(\omega)-y^{\prime}(\omega)\right|$ is not greater than $\gamma\left|y_{t}(\omega)\right|$ (a.s.). Clearly this implies that $y_{t+m}(\omega)$ is a (uniform) interior point of $X_{t+m}(\omega)$ (a.s.). In the financial context, (G4) typically holds for $m=1$ and essentially means a possibility of constructing a fully diversified portfolio $y_{t+m}$ starting from any non-zero portfolio, with proper estimates of the coordinates of the vector $y_{t+m}$ via the norm $\left|y_{t}\right|$ of the vector $y_{t}$ - compare with condition (G5) below.

The next result shows that under fairly general assumptions, any rapid path is asymptotically growth-optimal.

Theorem 3.4. If condition (G4) and condition (G2) with a constant $M_{t}=M$ independent of $t$ hold, then any rapid path is a.s. asymptotically growth-optimal.

Remark 1. Theorem 3.4 remains valid if condition $(\mathbf{G} 4)$ is replaced by the following one:

(G5) The cones $X_{t}(\omega)(t=0,1, \ldots)$ contain $\mathbb{R}_{+}^{N}$. There exist a real number $\gamma>0$ and a natural number $m$ such that for every $t \geq 0$ and every random vector $y_{t} \in \mathcal{X}_{t}$, there are random vectors $y_{t+1} \in \mathcal{X}_{t+1}, \ldots, y_{t+m} \in \mathcal{X}_{t+m}$, satisfying

$$
\left.\left(y_{t}, y_{t+1}\right) \in G_{t+1}(\omega), \ldots, \quad\left(y_{t+m-1}, y_{t+m}\right)\right) \in G_{t+m}(\omega) \quad \text { (a.s.) }
$$

and

$$
y_{t+m}(\omega) \geq \gamma e\left|y_{t}\right| \text { (a.s.). }
$$

For a proof of this assertion see Proposition 4.5 in Section 4 .

Let us formulate the main result pertaining to stationary (autonomous) systems. Let $T: \Omega \rightarrow \Omega$ be an automorphism of the given probability space $(\Omega, \mathcal{F}, P)$ such that conditions (1) hold. In the stationary framework, an important role is played by a class of paths called balanced. A path $x_{0}, x_{1}, x_{2}, \ldots$ is termed balanced if there exist an $\mathcal{F}_{0}$-measurable vector function $x(\omega)$ which is normalized by the condition $|x(\omega)|=1$ (a.s.) and an $\mathcal{F}_{1}$-measurable scalar function $\lambda(\omega)>0$ with $E|\ln \lambda(\omega)|<+\infty$ such that

$$
x_{0}(\omega)=x(\omega) ; x_{t}(\omega)=\lambda(\omega) \ldots \lambda\left(T^{t-1} \omega\right) x\left(T^{t} \omega\right), t \geq 1 .
$$

This definition expresses the idea of growth with stationary proportions and at a stationary rate. Clearly a pair of functions $x(\omega)$ and $\lambda(\omega)$, where $x \in \mathcal{X}_{0}$ and $\lambda(\omega)$ is an $\mathcal{F}_{1}$-measurable scalar function with $E|\ln \lambda(\omega)|<+\infty$, generates a balanced path if and only if $|x(\omega)|=1$ (a.s.) and

$$
(x(\omega), \lambda(\omega) x(T \omega)) \in G_{1}(\omega) \text { (a.s.). }
$$


A balanced path maximizing the expectation of the logarithm of the growth rate $E \ln \lambda(\omega)$ is called a von Neumann path. Note that condition (14) implies (by virtue of $(\mathbf{G 2}))$ that $\lambda(\omega)$ is essentially bounded.

A dual path $p_{1}, p_{2}, \ldots$ is called balanced if there exist an $\mathcal{F}_{1}$-measurable vector function $p(\omega)$ and an $\mathcal{F}_{1}$-measurable scalar function $\lambda(\omega)>0$ such that

$$
p_{1}(\omega)=p(\omega), p_{t}(\omega)=\frac{p\left(T^{t-1} \omega\right)}{\lambda(\omega) \ldots \lambda\left(T^{t-2} \omega\right)}, t=2,3, \ldots
$$

By virtue of (15) and using the invariance properties $G_{t+1}(\omega)=G_{t}(T \omega)$ and $\bar{p}_{t+1}=$ $T \bar{p}_{t}$ we can see that a function $p(\omega) \in L_{1}^{1}\left(\mathbb{R}^{N}\right)$ and an $\mathcal{F}_{1}$-measurable scalar function $\lambda(\omega)>0$ with $E|\ln \lambda(\omega)|<+\infty$ generate a balanced dual path if and only if for almost all $\omega$ we have:

$$
p(\omega) \in X_{0}^{*}(\omega), t=1,2, \ldots
$$

and

$$
\frac{E_{1} p(T \omega) b}{\lambda(\omega)} \leq p(\omega) a \text { for all }(a, b) \in G_{1}(\omega)
$$

A triplet of functions $(x, p, \lambda)$ forms a von Neumann equilibrium if the sequence $x_{0}, x_{1}, \ldots$ defined by $(13)$ is a balanced path and the sequence $p_{1}, p_{2}, \ldots$ defined by (15) is a dual balanced path supporting it.

Note that a triplet $(x, p, \lambda)$ is a von Neumann equilibrium if and only if the following conditions hold:

- $x(\omega)$ is a function in $\mathcal{X}_{0}$ normalized by the condition $|x(\omega)|=1$ (a.s.);

- $\lambda(\omega)>0$ is an $\mathcal{F}_{1}$-measurable scalar function with $E|\ln \lambda(\omega)|<+\infty$ satisfying (14) and maximizing $E \ln \lambda(\omega)$ among all balanced paths;

- $p(\omega) \in L_{1}^{1}\left(\mathbb{R}^{N}\right)$, and conditions (16), (17) and

$$
p(\omega) x(\omega)=1
$$

are fulfilled for almost all $\omega$.

Theorem 3.5. Suppose that conditions $(\boldsymbol{F})$ and $(\boldsymbol{G} \mathbf{1})-\left(\boldsymbol{G}_{4}\right)$ hold, the constants $M_{t}$, $\alpha_{t}, \theta_{t}, \Theta_{t}$ do not depend on $t$, and

$$
f_{t+1, k}(\omega)=f_{t, k}(T \omega), \hat{z}_{t+1}(\omega)=\hat{z}_{t}(T \omega) .
$$

Then a von Neumann equilibrium exists.

This result has the following important consequence. Let $(x, p, \lambda)$ be a von Neumann equilibrium, and consider the balanced path $\left(x_{t}\right)_{t=0}^{\infty}$ generated by the pair $(x, \lambda)$. By the definition of an equilibrium, it is rapid. Consequently (see Theorem 3.4), it is asymptotically growth-optimal. Thus, by virtue of Theorem 3.5, there exists a balanced path that is asymptotically growth-optimal in the class of all, not necessarily balanced paths! 


\section{General (non-stationary) model}

This section contains proofs of the results related to general (non-stationary) models of financial markets. We first establish Theorem 3.2 by deducing it from analogous results known for canonical von Neumann-Gale systems. At the end of the section, we prove Theorem 3.4 on the asymptotic optimality of rapid paths.

Let us assume that condition $(\mathbf{F})$ holds. A stochastic von Neumann-Gale dynamical system $\mathcal{G}$ defined by the state spaces $X_{t}(\omega)$ and the transition cones $G_{t}(\omega)$ generates a canonical von Neumann-Gale system $\mathcal{H}$ with the state spaces $\mathbb{R}_{+}^{K}$ and the transition cones

$$
H_{t}(\omega):=\left\{(c, d) \in \mathbb{R}_{+}^{K} \times \mathbb{R}_{+}^{K}:\left(F_{t-1}(\omega) c, F_{t}(\omega) d\right) \in G_{t}(\omega)\right\} .
$$

We will call $\mathcal{H}$ the canonical von Neumann-Gale dynamical system induced by $\mathcal{G}$.

Proposition 4.1. If $\mathcal{G}$ satisfies one of the conditions (G1)-(G3), then $\mathcal{H}$ satisfies one the following corresponding conditions.

(H1) For all $t \geq 1, \omega \in \Omega$ and $c \in \mathbb{R}_{+}^{K}$, the set $\left\{d:(c, d) \in H_{t}(\omega)\right\}$ is non-empty.

(H2) For each $t \geq 1$ there is a constant $M_{t}^{\prime}$ such that the set $H_{t}(\omega)$ is contained in $\left\{(c, d):|d| \leq M_{t}^{\prime}|c|\right\}$ for all $\omega \in \Omega$.

(H3) For every $t \geq 1$ there exist a strictly positive constant $\delta_{t}>0$ and a bounded vector function $\hat{w}_{t}(\omega)=\left(\hat{u}_{t-1}(\omega), \hat{v}_{t}(\omega)\right)$ such that $\hat{u}_{t-1}(\omega)$ is $\mathcal{F}_{t-1}$-measurable, $\hat{v}_{t}(\omega)$ is $\mathcal{F}_{t}$-measurable and $\mathbb{B}\left(\hat{w}_{t}(\omega), \delta_{t}\right) \subseteq H_{t}(\omega)$ for all $\omega$.

Proof. Suppose (G1) holds. Consider any $c \in \mathbb{R}_{+}^{K}$. Then $F_{t-1}(\omega) c \in X_{t-1}(\omega)$, and so there exists $b \in X_{t}(\omega)$ with $\left(F_{t-1}(\omega) c, b\right) \in G_{t}(\omega)$. Since $X_{t}(\omega)=F_{t}(\omega) \mathbb{R}_{+}^{K}$, there is $d \in \mathbb{R}_{+}^{K}$ such that $F_{t}(\omega) d=b$. Thus

$$
\left(F_{t-1}(\omega) c, F_{t}(\omega) d\right)=\left(F_{t-1}(\omega) c, b\right) \in G_{t}(\omega),
$$

and so $(c, d) \in H_{t}(\omega)$, which proves $(\mathbf{H 1})$.

Assume that condition (G2) is satisfied: $|b| \leq M_{t}|a|$ for all $(a, b) \in G_{t}(\omega)$. Let $(c, d) \in \mathbb{R}_{+}^{K} \times \mathbb{R}_{+}^{K}$ belong to $H_{t}(\omega)$, i.e. $\left(F_{t-1}(\omega) c, F_{t}(\omega) d\right) \in G_{t}(\omega)$. By virtue of $(8)$, we have $\left|F_{t-1}(\omega) c\right| \leq \Theta_{t-1}|c|$,

$$
\theta_{t}|d| \leq\left|F_{t}(\omega) d\right| \leq M_{t}\left|F_{t-1}(\omega) c\right| \leq M_{t} \Theta_{t-1}|c|,
$$

and so $|d| \leq M_{t}^{\prime}|c|$, which yields (H2) with the constant $M_{t}^{\prime}=\theta_{t}^{-1} M_{t} \Theta_{t-1}$.

Consider the vector function $\hat{z}_{t}(\omega)=\left(\hat{x}_{t-1}(\omega), \hat{y}_{t}(\omega)\right)$ involved in (G3). By using (7) and a measurable selection theorem (see Theorem 7.1 in the Appendix), we can construct an $\mathcal{F}_{t-1}$-measurable vector function $\hat{u}_{t-1}(\omega)$ and an $\mathcal{F}_{t}$-measurable vector function $\hat{v}_{t}(\omega)$ with values in $\mathbb{R}_{+}^{K}$ such that

$$
F_{t-1}(\omega) \hat{u}_{t-1}(\omega)=\hat{x}_{t-1}(\omega) \text { and } F_{t}(\omega) \hat{v}_{t}(\omega)=\hat{y}_{t}(\omega) .
$$

By virtue of the first inequality in (8), the function $\hat{w}_{t}(\omega)=\left(\hat{u}_{t-1}(\omega), \hat{v}_{t}(\omega)\right)$ is bounded. If $(c, d)$ is a vector in $\mathbb{R}_{+}^{K} \times \mathbb{R}_{+}^{K}$ such that $\left|(c, d)-\hat{w}_{t}(\omega)\right| \leq$ $\delta_{t}:=\alpha_{t} / \max \left\{\Theta_{t-1}, \Theta_{t}\right\}$, then $\left|\left(F_{t-1}(\omega) c, F_{t}(\omega) d\right)-\hat{z}_{t}(\omega)\right| \leq \alpha_{t}$. Consequently, $\left(F_{t-1}(\omega) c, F_{t}(\omega) d\right) \in G_{t}(\omega)$, and so $(c, d) \in H_{t}(\omega)$. Thus $\mathbb{B}\left(\hat{w}_{t}(\omega), \delta_{t}\right) \subseteq H_{t}(\omega)$, which proves (H3). 
To apply the results of the previous work to the dynamical system $\mathcal{H}$, we will need the following simple fact.

Remark 2. Condition (H3) implies the following one.

(h3) For every $t \geq 1$ there exist a strictly positive constant $\zeta_{t}>0$ and a bounded vector function $\hat{w}_{t}(\omega)=\left(\hat{u}_{t-1}(\omega), \hat{v}_{t}(\omega)\right)$ such that $\hat{u}_{t-1}(\omega)$ is $\mathcal{F}_{t-1}$-measurable, $\hat{v}_{t}(\omega)$ is $\mathcal{F}_{t}$-measurable, $\hat{w}_{t}(\omega) \in H_{t}(\omega)$ for all $\omega$, and $\hat{v}_{t}(\omega) \geq \zeta_{t} e$ (coordinate-wise) for all $\omega$, where $e=(1, \ldots, 1) \in \mathbb{R}_{+}^{K}$.

Observe that the function $\hat{w}_{t}(\omega)$ involved in (H3) has the properties described in (h3) with $\zeta_{t}=\delta_{t} / K$. Indeed, we have $\left|\zeta_{t} e\right|=\delta_{t}$. Thus $\left(\hat{u}_{t-1}(\omega), \hat{v}_{t}(\omega)-\zeta_{t} e\right) \in H_{t}(\omega)$, and so $\hat{v}_{t}(\omega)-\zeta_{t} e \in \mathbb{R}_{+}^{K}$, which means that $\hat{v}_{t}(\omega) \geq \zeta_{t} e$.

Let us examine relations between paths in $\mathcal{H}$ and $\mathcal{G}$.

Proposition 4.2. (a) If $\left(u_{t}\right)$ is a path in the dynamical system $\mathcal{H}$, then the sequence of functions $x_{t}(\omega)=F_{t}(\omega) u_{t}(\omega)$ forms a path $\left(x_{t}\right)$ in the dynamical system $\mathcal{G}$.

(b) If $\left(x_{t}\right)$ is a path in $\mathcal{G}$, then for each $t$ there exists an $\mathcal{F}_{t}$-measurable function $u_{t}(\omega)$ such that $x_{t}(\omega)=F_{t}(\omega) u_{t}(\omega)$. The sequence $\left(u_{t}\right)$ is a path in $\mathcal{H}$.

Proof. (a) The function $x_{t}(\omega)$ is $\mathcal{F}_{t}$-measurable, and $x_{t}(\omega) \in X_{t}(\omega)$ by virtue of (7). Furthermore, $x_{t}(\omega)$ is essentially bounded because $\left|F_{t}(\omega) u_{t}(\omega)\right| \leq \Theta_{t}\left|u_{t}(\omega)\right|$ (see (8)). Since $\left(u_{t-1}(\omega), u_{t}(\omega)\right) \in H_{t}(\omega)$ (a.s.), we have

$$
\left(F_{t-1}(\omega) u_{t-1}(\omega), F_{t}(\omega) u_{t}(\omega)\right) \in G_{t}(\omega) \text { (a.s.) }
$$

by the definition of $H_{t}(\omega)$. Thus $\left(x_{t}\right)$ is a path in $\mathcal{G}$.

(b) Let $\left(x_{t}\right)$ be a path in $\mathcal{G}$. Since $x_{t}(\omega) \in X_{t}(\omega)=F_{t}(\omega) \mathbb{R}_{+}^{K}$ (a.s.), by the measurable selection theorem there exists an $\mathcal{F}_{t}$-measurable function $u_{t}(\omega)$ with values in $\mathbb{R}_{+}^{K}$ such that $x_{t}(\omega)=F_{t}(\omega) u_{t}(\omega)$ (a.s.). In view of (8), we have $\left|u_{t}(\omega)\right| \leq\left|x_{t}(\omega)\right| \theta_{t}^{-1}$ (a.s.), so that $u_{t}$ is essentially bounded. Finally, we obtain

$$
\left(F_{t-1}(\omega) u_{t-1}(\omega), F_{t}(\omega) u_{t}(\omega)\right)=\left(x_{t-1}(\omega), x_{t}(\omega)\right) \in G_{t}(\omega) \text { (a.s.) }
$$

because $\left(x_{t}\right)$ is a path in $\mathcal{G}$. This means that $\left(u_{t-1}(\omega), u_{t}(\omega)\right) \in H_{t}(\omega)$ (a.s.), which proves that $\left(u_{t}\right)$ is a path in $\mathcal{H}$

The path $\left(x_{t}\right)$ in the dynamical system $\mathcal{G}$ defined by $x_{t}(\omega)=F_{t}(\omega) u_{t}(\omega)$ will be called the image of the path $\left(u_{t}\right)$ in $\mathcal{H}$. A key role in the proof of Theorem 3.2 is played by the following result.

Theorem 4.3. Let $\left(\tilde{u}_{t}\right)$ be a path in the dynamical system $\mathcal{H}$ and $\left(\tilde{x}_{t}\right)$ its image. If $\left(\tilde{u}_{t}\right)$ is rapid, then $\left(\tilde{x}_{t}\right)$ is rapid in $\mathcal{G}$.

Proof. Suppose $\tilde{u}_{0}, \tilde{u}_{1}, \ldots$ is a rapid path in the dynamical system $\mathcal{H}$, i.e., there exists a dual path $q_{1}, q_{2}, \ldots\left(q_{t} \in L_{t}^{1}\left(\mathbb{R}_{+}^{K}\right)\right)$ supporting it:

$$
q_{t+1}(\omega) \tilde{u}_{t}(\omega)=1 \text { (a.s.), } t \geq 0,
$$

and for almost all $\omega$,

$$
\bar{q}_{t+1}(\omega) d-q_{t}(\omega) c \leq 0,(c, d) \in H_{t}(\omega), t \geq 1,
$$


where $\bar{q}_{t+1}:=E_{t} q_{t+1}$. Define $\tilde{x}_{t}:=F_{t} \tilde{u}_{t}$. Let us show that $\left(\tilde{x}_{t}\right)$ is a rapid path in the dynamical system $\mathcal{G}$.

Fix some $t \geq 1$. By the definition of $H_{t}(\omega)$, a pair of vectors $(c, d) \in \mathbb{R}_{+}^{K} \times \mathbb{R}_{+}^{K}$ belongs to $H_{t}(\omega)$ if and only if $\left(F_{t-1}(\omega) c, F_{t}(\omega) d\right) \in G_{t}(\omega)$. Thus with probability 1 (for all $\omega$ in a set $\Omega_{1}$ of full measure), we have

$$
\bar{q}_{t+1}(\omega) d-q_{t}(\omega) c \leq 0,
$$

for any $(c, d) \in \mathbb{R}_{+}^{K} \times \mathbb{R}_{+}^{K}$ satisfying

$$
\left(F_{t-1}(\omega) c, F_{t}(\omega) d\right) \in G_{t}(\omega) .
$$

We will fix $\omega \in \Omega_{1}$ and omit it in the notation, if this does not lead to ambiguity. Since $\hat{z}_{t}=\left(\hat{x}_{t-1}, \hat{y}_{t}\right)=\left(F_{t-1} \hat{u}_{t-1}, F_{t} \hat{v}_{t}\right)$ is contained in $G_{t}(\omega)$ together with a ball of radius $\alpha_{t}$, we can apply the Kuhn-Tucker theorem (see Theorem 7.2 in the Appendix) to the convex set $X$ consisting of non-negative vectors $(c, d)$ in $\mathbb{R}_{+}^{K} \times \mathbb{R}_{+}^{K}$, the function $\Phi(c, d)=\bar{q}_{t+1} d-q_{t} c$, the cone $Z=G_{t}(\omega)$ and the mapping $R$ transforming $(c, d)$ into $\left(F_{t-1}(\omega) c, F_{t}(\omega) d\right) \in \mathbb{R}^{N} \times \mathbb{R}^{N}$. By using a measurable selection argument, we construct $\mathcal{F}_{t}$-measurable functions $a_{t}(\omega)$ and $b_{t}(\omega)$ taking values in $\mathbb{R}^{N}$ such that for all $\omega \in \Omega_{1}$,

$$
\begin{gathered}
b_{t} b-a_{t} a \leq 0,(a, b) \in G_{t}(\omega), \\
\bar{q}_{t+1} d-q_{t} c-\left[b_{t} F_{t} d-a_{t} F_{t-1} c\right] \leq 0,(c, d) \in \mathbb{R}_{+}^{K} \times \mathbb{R}_{+}^{K},
\end{gathered}
$$

and

$$
\left|b_{t}\right|+\left|a_{t}\right| \leq 2 N \alpha_{t}^{-1}\left(q_{t} \hat{u}_{t-1}-\bar{q}_{t+1} \hat{v}_{t}\right) .
$$

Let us show that the sequence of vector functions

$$
p_{t}(\omega):=a_{t}(\omega), t \geq 1
$$

forms a dual path in the dynamical system $\mathcal{G}$ supporting the path $\left(\tilde{x}_{t}\right)$. The fact that $E\left|a_{t}(\omega)\right|<\infty$ follows from (20). By setting first $c=0$ and then $d=0$ in (19), we get

$$
\begin{aligned}
& \bar{q}_{t+1} d \leq b_{t} F_{t} d, d \in \mathbb{R}_{+}^{K}, t \geq 1, \\
& q_{t} c \geq a_{t} F_{t-1} c, c \in \mathbb{R}_{+}^{K}, t \geq 1 .
\end{aligned}
$$

For all pairs $(x, y)$ of functions in $L_{t}^{\infty}\left(\mathbb{R}^{N}\right) \times L_{t}^{\infty}\left(\mathbb{R}^{N}\right)$ with $(x, y) \in G_{t}(\omega)$ (a.s.), there exist $\mathcal{F}_{t}$-measurable vector functions $u(\omega)$ and $v(\omega)$ taking values in $\mathbb{R}_{+}^{K}$, such that $y=F_{t} v, x=F_{t-1} u$ and $(u, v) \in H_{t}(\omega)$ (a.s.). The functions $u$ and $v$ are essentially bounded by virtue of the first inequality in (8). Therefore

$$
\begin{aligned}
E p_{t} x & =E a_{t} x \geq E b_{t} y=E b_{t} F_{t} v \\
& \geq E \bar{q}_{t+1} v=E q_{t+1} v \geq E a_{t+1} F_{t} v=E p_{t+1} y,
\end{aligned}
$$

which yields (3) for $t \geq 1$. In this chain of relations, the first inequality holds because $b_{t} y \leq a_{t} x$ (a.s.) as long as $(x, y) \in G_{t}(\omega)$ (a.s.), which follows from (18). The second 
inequality is a consequence of (21) and the third follows from (22) with $t+1$ in place of $t$.

For $t \geq 0$ we have

$$
p_{t+1} \tilde{x}_{t}=a_{t+1} \tilde{x}_{t}=a_{t+1} F_{t} \tilde{u}_{t} \leq q_{t+1} \tilde{u}_{t}=1 \text { (a.s.) }
$$

where the inequality follows from (22) with $t$ replaced by $t+1$. Furthermore, we get

$$
\begin{aligned}
E p_{t+1} \tilde{x}_{t} & =E a_{t+1} \tilde{x}_{t} \geq E b_{t+1} \tilde{x}_{t+1}=E b_{t+1} F_{t+1} \tilde{u}_{t+1} \\
& \geq E \bar{q}_{t+2} \tilde{u}_{t+1}=E q_{t+2} \tilde{u}_{t+1}=1 .
\end{aligned}
$$

In this chain of relations, the first inequality holds because $\left(\tilde{x}_{t}, \tilde{x}_{t+1}\right) \in G_{t+1}(\omega)$ (a.s.) and the second follows from (21) with $t+1$ in place of $t$. By combining (23) and (24), we conclude that $p_{t+1} \tilde{x}_{t}=1$ (a.s.).

Let us show that for all $t \geq 1$ and almost all $\omega \in \Omega$,

$$
p_{t}(\omega) a \geq 0 \text { for } a \in X_{t-1}(\omega),
$$

i.e. $p_{t}(\omega) \in X_{t-1}^{*}(\omega)$ (a.s.). Denote by $\Omega_{t}$ the set of those $\omega$ for which $b_{t} F_{t} \geq 0$. By virtue of $(21)$, we have $P\left(\Omega_{t}\right)=1$. Fix any $\omega \in \Omega_{t}, a \in X_{t-1}(\omega)$ and consider some $c \in \mathbb{R}_{+}^{K}$ for which $F_{t-1}(\omega) c=a$. In view of $(\mathbf{H 1})$, there exists $d \in \mathbb{R}_{+}^{K}$ such that $(c, d) \in H_{t}(\omega)$. Then $b_{t} F_{t} d \leq a_{t} F_{t-1} c$ by virtue of (18). From this we get

$$
p_{t} a=a_{t} F_{t-1} c \geq b_{t} F_{t} d \geq 0,
$$

which completes the proof.

The following results in the case of a canonical von Neumann-Gale dynamical system are obtained in [5], Theorem 1.

Theorem 4.4. Let conditions $(\boldsymbol{H} 1)-(\boldsymbol{H} 3)$ hold. Let $u_{0}(\omega)$ be a vector function in $L_{0}^{\infty}\left(\mathbb{R}^{K}\right)$ such that $u_{0}(\omega) \geq \delta$ for some constants $\delta>0$. Then the following assertions are valid.

(i) For each $n \geq 1$, there exists a finite rapid path of length $n$ with the initial state $u_{0}(\omega)$.

(ii) There exists an infinite rapid path with the initial state $u_{0}(\omega)$.

Proof of Theorem 3.2. Let $x_{0}$ be a function in $\mathcal{X}_{0}$ such that $\mathbb{B}\left(x_{0}(\omega), \varepsilon\right) \subseteq X_{0}(\omega)$ (a.s.), where $\varepsilon$ is a strictly positive constant. By virtue of (7) and the measurable selection theorem, there exists an $\mathcal{F}_{0}$-measurable function $u_{0}(\omega)$ with values in $\mathbb{R}_{+}^{K}$ such that $x_{0}(\omega)=F_{0}(\omega) u_{0}(\omega)$ (a.s.). By virtue of the first inequality in (8), the function $u_{0}(\omega)$ is bounded. Let us prove $u_{0}(\omega) \geq \delta e$, where $\delta=\varepsilon / K$ and $e=(1, \ldots, 1) \in \mathbb{R}_{+}^{K}$. To this end, we define $y(\omega)=F_{0}(\omega) \delta e$ and observe that

$$
|y(\omega)|=\delta\left|F_{0}(\omega) e\right|=\delta\left|\sum_{k=1}^{K} f_{0, k}(\omega)\right| \leq \varepsilon,
$$

where the inequality follows form the fact we can assume that $f_{0, k}(\omega), k=$ $1,2, \ldots, K, \omega \in \Omega$ are normalized. Then $x_{0}(\omega)-y(\omega) \in X_{0}(\omega)$ (a.s.), and so there 
exists an $\mathcal{F}_{0}$-measurable function $h(\omega)$ with values in $\mathbb{R}_{+}^{K}$ such that

$$
F_{0}(\omega) u_{0}(\omega)-F_{0}(\omega) \delta e=F_{0}(\omega) h(\omega) .
$$

Then $u_{0}(\omega)=\delta e+h(\omega)$, which means $u_{0}(\omega) \geq \delta e$. Here, we use the fact that $F_{0}(\omega)$ is the one-to-one operator, which follows from (8).

By virtue of Proposition 4.1, conditions (H1)-(H3) hold. Then by assertion (i) of Theorem 4.4, for each $n \geq 1$, there exists a finite rapid path $\left(u_{t}\right)_{t=0}^{n}$ of length $n$ with the initial state $u_{0}(\omega)$. Let $x_{t}=F_{t} u_{t}$. By virtue of Theorem 4.3, $\left(x_{t}\right)_{t=0}^{n}$ is the rapid path of length $n$ in the dynamical system $\mathcal{G}$ with the initial state $x_{0}(\omega)$. Further, by assertion (ii) of Theorem 4.4, there exists an infinite rapid path $\left(u_{t}\right)$ with the initial state $u_{0}(\omega)$. If we put $x_{t}=F_{t} u_{t}$ and use Theorem 4.3 , we conclude that $\left(x_{t}\right)$ is the infinite rapid path in $\mathcal{G}$. The proof is complete.

Proof of Theorem 3.4. Let $x_{0}, x_{1}, \ldots$ be a rapid path supported by a dual path $p_{1}, p_{2}, \ldots$ For all $y \in L_{t+m}^{\infty}\left(\mathbb{R}^{N}\right)$ with $|y(\omega)| \leq \gamma\left|y_{t}(\omega)\right|$, by using (2) and (11), we have

$$
p_{t+m} y_{t+m-1} \geq \bar{p}_{t+m+1}\left(y_{t+m}+y\right)=\bar{p}_{t+m+1} y_{t+m}+\bar{p}_{t+m+1} y \geq \bar{p}_{t+m+1} y
$$

because $\bar{p}_{t+m+1} y_{t+m} \geq 0$ (a.s.). The last inequality is valid since $y_{t+m}(\omega) \in X_{t+m}(\omega)$ and $p_{t+m+1}(\omega) \in X_{t+m}^{*}(\omega)$ (a.s.), which yields $p_{t+m+1} y_{t+m} \geq 0$ (a.s.) and so $\bar{p}_{t+m+1} y_{t+m} \geq 0$ (a.s.). Put

$$
y=\frac{\bar{p}_{t+m+1}}{\left|\bar{p}_{t+m+1}\right|} \gamma\left|y_{t}\right|
$$

Then $|y(\omega)|=\gamma\left|y_{t}(\omega)\right|$ and $y \in \mathcal{X}_{t+m}$. Consequently, (25) can be applied to $y$ defined by (26). Observe that

$$
\bar{p}_{t+m+1} y=\frac{|| \bar{p}_{t+m+1}||^{2}}{\left|\bar{p}_{t+m+1}\right|} \gamma\left|y_{t}\right| \geq\left|\bar{p}_{t+m+1}\right| N^{-1} \gamma\left|y_{t}\right|
$$

where $\|\cdot\|$ is the Euclidean norm in $\mathbb{R}^{N}$ (note that $\|\cdot\| \geq|\cdot| / \sqrt{N}$ ). Further, the equality $p_{t+m+1} x_{t+m}=1$ implies $\bar{p}_{t+m+1} x_{t+m}=1$, and so

$$
\left|\bar{p}_{t+m+1}\right|\left|x_{t+m}\right| \geq 1 \text {, }
$$

and it follows from $(\mathbf{G 2})$ with a constant $M_{t}=M$ independent of $t$ that

$$
\left|x_{t+m}\right| \leq M^{m}\left|x_{t}\right|
$$

By combining (28) and (29), we get

$$
\left|\bar{p}_{t+m+1}\right| \geq M^{-m}\left|x_{t}\right|^{-1},
$$

and by using (27), (25) and (30), we obtain

$$
p_{t+m} y_{t+m-1} \geq M^{-m} N^{-1} \gamma\left|y_{t}\right|\left|x_{t}\right|^{-1},
$$


which yields

$$
p_{t+1} y_{t} \geq E_{t+1} p_{t+m+1} y_{t+m-1} \geq M^{-m} N^{-1} \gamma\left|y_{t}\right|\left|x_{t}\right|^{-1} .
$$

Since $p_{t+1} y_{t}$ is a non-negative supermartingale, the proof is complete.

We provide a version of Theorem 3.4 in which $(\mathbf{G} 4)$ is replaced by another assumption.

Proposition 4.5. Theorem 3.4 remains valid if condition (G4) is replaced by (G5).

Proof. Let $x_{0}, x_{1}, \ldots$ be a rapid path supported by a dual path $p_{1}, p_{2}, \ldots$. Since $X_{t}(\omega) \supseteq$ $\mathbb{R}_{+}^{N}$, any $p_{t+1}(\omega) \in X_{t}^{*}(\omega)$ is non-negative (a.s.). By using this, (2), (12) and the fact that $\left|p_{t+m+1}\right| \geq M^{-m}\left|x_{t}\right|^{-1}$, which follows from the equality $p_{t+m+1} x_{t+m}=1$ and (G2) with a constant $M_{t}=M$ independent of $t$, we get

$$
\begin{aligned}
p_{t+1} y_{t} & \geq E_{t+1} p_{t+m+1} y_{t+m} \geq \gamma E_{t+1}\left|p_{t+m+1}\right|\left|y_{t}\right| \\
& \geq \gamma E_{t+1}\left(M^{-m}\left|x_{t}\right|^{-1}\right)\left|y_{t}\right|=\gamma M^{-m}\left|x_{t}\right|^{-1}\left|y_{t}\right| .
\end{aligned}
$$

Thus $\left|x_{t}\right|^{-1}\left|y_{t}\right|$ is dominated by a non-negative supermartingale $p_{t+1} y_{t}$, which completes the proof.

\section{Stationary Model}

In the case of a stationary system, let us assume that conditions $(\mathbf{F})$ and $(\mathbf{G 1})-(\mathbf{G} 4)$ hold and suppose, additionally, that

$$
f_{t+1, k}(\omega)=f_{t, k}(T \omega)
$$

$\hat{z}_{t+1}(\omega)=\hat{z}_{t}(T \omega)$, and the constants $M_{t}, \alpha_{t}, \theta_{t}$ and $\Theta_{t}$ do not depend on $t$. Clearly (31) implies that $X_{t+1}(\omega)=x_{t}(T \omega)$. Let us formulate analogues of the conditions (G1)-(G4) in the case of a stationary model as follows.

$\left(\mathbf{G 1}^{\prime}\right)$ For all $\omega \in \Omega$ and $a \in X_{0}(\omega)$, the set $\left\{b:(a, b) \in G_{1}(\omega)\right\}$ is non-empty.

$\left(\mathbf{G 2}^{\prime}\right)$ There is a constant $M$ such that the set $G_{1}(\omega)$ is contained in $\{(a, b):|b| \leq$ $M|a|\}$ for all $\omega \in \Omega$.

$\left(\mathbf{G 3}^{\prime}\right)$ There exist a strictly positive constant $\alpha>0$ and a bounded vector function $\hat{z}_{1}(\omega)=\left(\hat{x}_{0}(\omega), \hat{y}_{1}(\omega)\right)$ such that $\hat{x}_{0}(\omega)$ is $\mathcal{F}_{0}$-measurable, $\hat{y}_{1}(\omega)$ is $\mathcal{F}_{1}$-measurable and $\mathbb{B}\left(\hat{z}_{1}(\omega), \alpha\right) \subseteq G_{1}(\omega)$ for all $\omega$.

$\left(\mathbf{G} 4^{\prime}\right)$ There exists a natural number $m$ such that for every random state $y_{0} \in \mathcal{X}_{0}$, one can find a real number $\gamma>0$ and random states $y_{1} \in \mathcal{X}_{1}, \ldots, y_{m} \in \mathcal{X}_{m}$, satisfying with probability one

$$
\left(y_{0}(\omega), y_{1}(\omega)\right) \in G_{1}(\omega), \ldots, \quad\left(y_{m-1}(\omega), y_{m}(\omega)+y(\omega)\right) \in G_{m}(\omega)
$$

for all $y \in L_{m}^{\infty}\left(\mathbb{R}^{N}\right)$ with $|y(\omega)| \leq \gamma\left|y_{0}(\omega)\right|$.

Observe that the above conditions are versions of conditions $(\mathbf{G} \mathbf{1})-(\mathbf{G} 4)$ formulated for one moment of time $t=0$. Clearly in the stationary case, these requirements hold for some $t$ if and only if they hold for each $t$. 
Proposition 5.1. If $\mathcal{G}$ satisfies one of the conditions $\left(\boldsymbol{G} \mathbf{1}^{\prime}\right)-\left(G_{4}{ }^{\prime}\right)$, then $\mathcal{H}$ satisfies one of the following corresponding conditions.

(H1') For all $\omega \in \Omega$ and $c \in \mathbb{R}_{+}^{K}$, the set $\left\{d:(c, d) \in H_{1}(\omega)\right\}$ is non-empty.

$\left(\boldsymbol{H} 2^{\prime}\right)$ There is a constant $M^{\prime}$ such that the set $H_{1}(\omega)$ is contained in $\{(c, d):|d| \leq$ $\left.M^{\prime}|c|\right\}$ for all $\omega \in \Omega$.

(Hз') There exist a strictly positive constant $\delta>0$ and a bounded vector function $\hat{w}_{1}(\omega)=\left(\hat{u}_{0}(\omega), \hat{v}_{1}(\omega)\right)$ such that $\hat{u}_{0}(\omega)$ is $\mathcal{F}_{0}$-measurable, $\hat{v}_{1}(\omega)$ is $\mathcal{F}_{1}$-measurable and $\mathbb{B}\left(\hat{w}_{1}(\omega), \delta\right) \subseteq H_{1}(\omega)$ for all $\omega$.

(H4') There exists a natural number $m$ such that for every non-negative function $u_{0} \in L_{0}^{\infty}\left(\mathbb{R}^{K}\right)$, one can find a real number $\gamma^{\prime}>0$ and non-negative vector functions $u_{1} \in L_{1}^{\infty}\left(\mathbb{R}^{K}\right), \ldots, u_{m} \in L_{m}^{\infty}\left(\mathbb{R}^{K}\right)$, satisfying with probability one

$$
\left(u_{0}(\omega), u_{1}(\omega)\right) \in H_{1}(\omega), \ldots, \quad\left(u_{m-1}(\omega), u_{m}(\omega)+u(\omega)\right) \in H_{m}(\omega)
$$

for all $u \in L_{m}^{\infty}\left(\mathbb{R}^{K}\right)$ with $|u(\omega)| \leq \gamma^{\prime}\left|u_{0}(\omega)\right|$.

Proof. If one of the conditions $\left(\mathbf{G 1}^{\prime}\right)-\left(\mathbf{G 3}^{\prime}\right)$ holds, then the analogous condition holds. The proof is exactly the same as the proof of Proposition 4.1, with the additional assumption that the constants $M_{t}, \alpha_{t}, \theta_{t}$ and $\Theta_{t}$ do not depend on $t$.

Suppose $\left(\mathbf{G} 4^{\prime}\right)$ holds. Consider any non-negative vector function $u_{0} \in L_{0}^{\infty}\left(\mathbb{R}^{K}\right)$. Let $y_{0}(\omega)=F_{0}(\omega) u_{0}(\omega)$. The function $y_{0}(\omega)$ is $\mathcal{F}_{0}$-measurable, and $y_{0}(\omega) \in X_{0}(\omega)$ by virtue of (7). Furthermore, $y_{0}(\omega)$ is essentially bounded because $\left|F_{0}(\omega) u_{0}(\omega)\right| \leq$ $\Theta\left|u_{0}(\omega)\right|$ (see (8)). Then by virtue of $\left(\mathbf{G} \mathbf{4}^{\prime}\right)$ there exist a natural number $m$, a real number $\gamma>0$ and random states $y_{1} \in \mathcal{X}_{1}, \ldots, y_{m} \in \mathcal{X}_{m}$, satisfying (32) with probability one for all $y \in L_{m}^{\infty}\left(\mathbb{R}^{K}\right)$ with $|y(\omega)| \leq \gamma\left|y_{0}(\omega)\right|$. Since for every $1 \leq t \leq m, y_{t}(\omega) \in$ $X_{t}(\omega)=F_{t}(\omega) \mathbb{R}_{+}^{K}$ (a.s.), by the measurable selection theorem, there exists an $\mathcal{F}_{t^{-}}$ measurable function $u_{t}(\omega)$ with values in $\mathbb{R}_{+}^{K}$ such that $y_{t}(\omega)=F_{t}(\omega) u_{t}(\omega)$ (a.s.). In view of (8), the function $u_{t}$ is essentially bounded. Thus for every $0 \leq t \leq m-2$, we obtain

$$
\left(F_{t}(\omega) u_{t}(\omega), F_{t+1}(\omega) u_{t+1}(\omega)\right)=\left(y_{t}(\omega), y_{t+1}(\omega)\right) \in G_{t+1}(\omega) \text { (a.s.), }
$$

which yields $\left(u_{t}(\omega), u_{t+1}(\omega)\right) \in H_{t+1}(\omega)$ (a.s.). Put $\gamma^{\prime}=K^{-1} \Theta^{-1} \theta \gamma$ and consider $u \in L_{m}^{\infty}\left(\mathbb{R}^{K}\right)$ with $|u(\omega)| \leq \gamma^{\prime}\left|u_{0}(\omega)\right|$. We wish to prove

$$
\left(u_{m-1}(\omega), u_{m}(\omega)+u(\omega)\right) \in H_{m}(\omega) \text { (a.s.). }
$$

To this end, let us first prove that $u_{m}(\omega)+u(\omega) \in \mathbb{R}_{+}^{K}$. If we put $y(\omega)=$ $-\gamma^{\prime}\left|u_{0}(\omega)\right| F_{m}(\omega) e$, then we have

$$
|y(\omega)| \leq K \Theta \gamma^{\prime}\left|u_{0}(\omega)\right| \leq K \Theta \gamma^{\prime} \theta^{-1}\left|y_{0}(\omega)\right|=\gamma\left|y_{0}(\omega)\right|,
$$

where both inequalities follow from (8) and the fact that $y \in L_{m}^{\infty}\left(\mathbb{R}^{N}\right)$. Thus, $\left(y_{m-1}(\omega), y_{m}(\omega)+y(\omega)\right) \in G_{m}(\omega)$ and so there exists an $\mathcal{F}_{m}$-measurable function $h(\omega) \in \mathbb{R}_{+}^{K}$ such that $F_{m}(\omega) h(\omega)=y_{m}(\omega)+y(\omega)$. As a consequence, we have

$$
F_{m}(\omega) u_{m}(\omega)=F_{m}(\omega)\left(h(\omega)+\gamma^{\prime}\left|u_{0}(\omega)\right| e\right),
$$


and so $u_{m}(\omega)=h(\omega)+\gamma^{\prime}\left|u_{0}(\omega)\right| e$, which yields $u_{m}(\omega) \geq \gamma^{\prime}\left|u_{0}(\omega)\right| e$. Consequently,

$$
u_{m}(\omega)+u(\omega) \geq \gamma^{\prime}\left|u_{0}(\omega)\right| e+u(\omega) \geq|u(\omega)| e+u(\omega) \geq 0 .
$$

Now put $y(\omega)=F_{m}(\omega)\left(u_{m}(\omega)+u(\omega)\right)-F_{m}(\omega) u_{m}(\omega)$. Observe that

$$
\begin{aligned}
|y(\omega)| & \leq \Theta\left|u_{m}(\omega)+u(\omega)-u_{m}(\omega)\right|=\Theta|u(\omega)| \leq \Theta \gamma^{\prime}\left|u_{0}(\omega)\right| \\
& \leq \Theta \gamma^{\prime} \theta^{-1}\left|y_{0}(\omega)\right|=K^{-1} \gamma\left|y_{0}(\omega)\right| \leq \gamma\left|y_{0}(\omega)\right|,
\end{aligned}
$$

and $y \in L_{m}^{\infty}\left(\mathbb{R}^{N}\right)$. Thus we have

$$
\begin{aligned}
\left(F_{m-1}(\omega) u_{m-1}(\omega), F_{m}(\omega)\left(u_{m}(\omega)+\right.\right. & u(\omega))) \\
& =\left(y_{m-1}(\omega), y_{m}(\omega)+y(\omega)\right) \in G_{m}(\omega) \text { (a.s.) }
\end{aligned}
$$

which means that $\left(u_{m-1}(\omega), u_{m}(\omega)+u(\omega)\right) \in H_{m}(\omega)$ (a.s.).

To apply the results of the previous work to the dynamical system $\mathcal{H}$, we will need the following fact.

Remark 3. Condition $\left(\mathbf{H} \mathbf{4}^{\prime}\right)$ implies the following one.

$\left(\mathbf{h} 4^{\prime}\right)$ There exists a natural number $m$ such that for every non-negative function $u_{0} \in L_{0}^{\infty}\left(\mathbb{R}^{K}\right)$, one can find a real number $\rho>0$ and non-negative vector functions $u_{1} \in L_{0}^{\infty}\left(\mathbb{R}^{K}\right), \ldots, u_{m} \in L_{m}^{\infty}\left(\mathbb{R}^{K}\right)$, satisfying with probability one

$$
\left(u_{0}(\omega), u_{1}(\omega)\right) \in H_{1}(\omega), \ldots, \quad\left(u_{m-1}(\omega), u_{m}(\omega)\right) \in H_{m}(\omega)
$$

and

$$
u_{m}(\omega) \geq \rho e\left|u_{0}\right|,
$$

where $e=(1, \ldots, 1) \in \mathbb{R}_{+}^{K}$.

Observe that the functions $u_{1}, \ldots, u_{m}$ involved in $\left(\mathbf{H} \mathbf{4}^{\prime}\right)$ have the properties described in $\left(\mathbf{h} 4^{\prime}\right)$ with $\rho=\gamma^{\prime} / K$. This is so because, if $u_{m}(\omega)+u(\omega) \in \mathbb{R}_{+}^{K}$ for all $u \in L_{m}^{\infty}\left(\mathbb{R}^{K}\right)$ with $|u(\omega)| \leq \gamma^{\prime}\left|u_{0}(\omega)\right|$ then $u_{m}(\omega) \geq e \rho\left|u_{0}\right|$. Indeed, the inequality $u_{m}(\omega) \geq \rho e\left|u_{0}(\omega)\right|$ can be written as $u_{m}(\omega)+v(\omega) \in \mathbb{R}_{+}^{K}$ where $v:=-\rho e\left|u_{0}\right|$ and $|v(\omega)|=\gamma^{\prime}\left|u_{0}(\omega)\right|$.

A key role in the proof of Theorem 3.5 is played by the following result.

Theorem 5.2. Let $\left(u_{0}, q_{1}, \lambda_{1}\right)$ be a triplet of functions forming a von Neumann equilibrium in the dynamical system $\mathcal{H}$. Then there exists a triplet $\left(\tilde{x}_{0}, \tilde{p}_{1}, \tilde{\lambda}_{1}\right)$ forming a von Neumann equilibrium in the dynamical system $\mathcal{G}$.

Proof. Suppose $\left(u_{0}, q_{1}, \lambda_{1}\right)$ is a triplet of functions forming a von Neumann equilibrium in the dynamical system $\mathcal{H}$. Then for almost all $\omega$, we have $q_{1}(\omega) \in L_{1}^{1,+}\left(\mathbb{R}^{K}\right)$,

$$
\left(u_{0}(\omega), \lambda_{1}(\omega) u_{1}(\omega)\right) \in H_{1}(\omega), \quad q_{1}(\omega) u_{0}(\omega)=1,
$$

and

$$
\frac{\bar{q}_{2}(\omega) d}{\lambda_{1}(\omega)}-q_{1}(\omega) c \leq 0,(c, d) \in H_{1}(\omega)
$$


where $u_{1}=T u_{0}, q_{2}=T q_{1}$ and $\bar{q}_{2}=E_{1} q_{2}$. By the definition of $H_{1}(\omega)$, a pair of vectors $(c, d) \in \mathbb{R}_{+}^{K} \times \mathbb{R}_{+}^{K}$ belongs to $H_{1}(\omega)$ if and only if $\left(F_{0}(\omega) c, F_{1}(\omega) d\right) \in G_{1}(\omega)$. Thus with probability 1 (for all $\omega$ in a set $\Omega_{1}$ of full measure), we have

$$
\frac{\bar{q}_{2}(\omega) d}{\lambda_{1}(\omega)}-q_{1}(\omega) c \leq 0
$$

for any $(c, d) \in \mathbb{R}_{+}^{K} \times \mathbb{R}_{+}^{K}$ satisfying

$$
\left(F_{0}(\omega) c, F_{1}(\omega) d\right) \in G_{1}(\omega) .
$$

We will fix $\omega \in \Omega_{1}$ and omit it in the notation, if this does not lead to ambiguity. Since $\hat{z}_{1}=\left(\hat{x}_{0}, \hat{y}_{1}\right)=\left(F_{0} \hat{u}_{0}, F_{1} \hat{v}_{1}\right)$ is contained in $G_{1}(\omega)$ together with a ball of radius $\alpha$, we can apply the Kuhn-Tucker theorem (see Theorem 7.2 in the Appendix) to the convex set $X$ consisting of non-negative vectors $(c, d)$ in $\mathbb{R}_{+}^{K} \times \mathbb{R}_{+}^{K}$, the function

$$
\Phi(c, d)=\bar{q}_{2} d / \lambda_{1}-q_{1} c,
$$

the cone $Z=G_{1}(\omega)$ and the mapping $R$ transforming $(c, d)$ into $\left(F_{0}(\omega) c, F_{1}(\omega) d\right) \in$ $\mathbb{R}^{N} \times \mathbb{R}^{N}$. By using a measurable selection argument, we construct $\mathcal{F}_{1}$-measurable functions $a_{1}(\omega)$ and $b_{1}(\omega)$ taking values in $\mathbb{R}^{N}$ such that for all $\omega \in \Omega_{1}$,

$$
b_{1} b-a_{1} a \leq 0,(a, b) \in G_{1}(\omega),
$$

for all $(c, d) \in \mathbb{R}_{+}^{K} \times \mathbb{R}_{+}^{K}$,

$$
\frac{\bar{q}_{2} d}{\lambda_{1}}-q_{1} c-\left[b_{1} F_{1} d-a_{1} F_{0} c\right] \leq 0
$$

and

$$
\left|b_{1}\right|+\left|a_{1}\right| \leq 2 N \alpha^{-1}\left(q_{1} \hat{u}_{0}-\frac{\bar{q}_{2} \hat{v}_{1}}{\lambda_{1}}\right)
$$

Define $x_{0}:=F_{0} u_{0}$. The function $x_{0}(\omega)=F_{0}(\omega) u_{0}(\omega)$ is $\mathcal{F}_{0}$-measurable and $x_{0} \in \mathcal{X}_{0}$ by virtue of (7). Furthermore, $x_{0}(\omega)$ is essentially bounded. Put

$$
x_{1}=T x_{0}, \tilde{x}_{0}=x_{0} /\left|x_{0}\right|, \tilde{x}_{1}=T \tilde{x}, \text { and } \tilde{\lambda}_{1}=\lambda_{1}\left|x_{1}\right| /\left|x_{0}\right| .
$$

Let us show that the triplet $\left(\tilde{x}_{0}, \tilde{p}_{1}, \tilde{\lambda}_{1}\right)$ is an equilibrium in $\mathcal{G}$. We have $\tilde{x}_{0} \in \mathcal{X}_{0}$ and $\left|\tilde{x}_{0}\right|=1$. Since $\left|u_{0}\right|=1$ (a.s.), in view of (8) we have $\theta \leq\left|x_{0}\right|=\left|F_{0}(\omega) u_{0}\right| \leq \Theta$, and so $E|\ln | x_{0}||<\infty$. Therefore

$$
E \ln \tilde{\lambda}_{1}=E \ln \lambda_{1}+E \ln \left|x_{1}\right|-E \ln \left|x_{0}\right|=E \ln \lambda_{1} .
$$

Further, we have

$$
\left(\tilde{x}_{0}, \tilde{\lambda}_{1} \tilde{x}_{1}\right)=\left(\frac{x_{0}}{\left|x_{0}\right|}, \frac{\lambda_{1}\left|x_{1}\right|}{\left|x_{0}\right|} \frac{x_{1}}{\left|x_{1}\right|}\right)=\left(\frac{x_{0}}{\left|x_{0}\right|}, \frac{\lambda_{1} x_{1}}{\left|x_{0}\right|}\right)
$$




$$
=\left|x_{0}\right|^{-1}\left(F_{0} u_{0}, \lambda_{1} F_{1}\left(u_{1}\right)\right)=\left|x_{0}\right|^{-1}\left(F_{0} u_{0}, F_{1}\left(\lambda_{1} u_{1}\right)\right) \in G_{1}(\omega) \text { (a.s.). }
$$

Thus the pair of functions $\tilde{x}_{0}$ and $\tilde{\lambda}_{1}$ generates a balanced path.

Put $\tilde{p}_{1}:=\left|x_{0}\right| a_{1}$. We can see from (35) that $E\left|a_{1}(\omega)\right|<\infty$, and so $E\left|\tilde{p}_{1}(\omega)\right|<\infty$. By setting first $c=0$ and then $d=0$ in (34), we get

$$
\begin{aligned}
& \frac{\bar{q}_{2}}{\lambda_{1}} \leq b_{1} F_{1}, \\
& q_{1} \geq a_{1} F_{0} .
\end{aligned}
$$

By applying the operator $T$ to both sides of inequality (38), we get

$$
q_{2} \geq a_{2} F_{1}
$$

where $a_{2}:=T a_{1}$. For all pairs $(x, y)$ of functions in $L_{1}^{\infty}\left(\mathbb{R}^{N}\right) \times L_{1}^{\infty}\left(\mathbb{R}^{N}\right)$ such that $(x, y) \in G_{1}(\omega)$ (a.s.), there exist $\mathcal{F}_{1}$-measurable vector functions $u$ and $v$ taking values in $\mathbb{R}_{+}^{K}$, such that $x=F_{0} u, y=F_{1} v$ and $(u, v) \in H_{1}(\omega)$ (a.s.). These functions are essentially bounded in view of the first inequality in (8). By setting $\tilde{p}_{2}=T \tilde{p}_{1}$, we get

$$
\begin{aligned}
E \tilde{p}_{1} x & =E\left|x_{0}\right| a_{1} x \geq E\left|x_{0}\right| b_{1} y=E\left|x_{0}\right| b_{1} F_{1} v \geq E \frac{\bar{q}_{2}\left|x_{0}\right| v}{\lambda_{1}} \\
& =E \frac{q_{2}\left|x_{0}\right| v}{\lambda_{1}} \geq E \frac{a_{2} F_{1}\left|x_{0}\right| v}{\lambda_{1}}=E \frac{\left|x_{0}\right| a_{2} y}{\lambda_{1}}=E \frac{\tilde{p}_{2} y}{\tilde{\lambda}_{1}}=E \frac{E_{1}\left(\tilde{p}_{2}\right) y}{\tilde{\lambda}_{1}} .
\end{aligned}
$$

In this chain of relations, the first inequality holds because $b_{1} y\left|x_{0}\right| \leq a_{1} x\left|x_{0}\right|$ (a.s.) as long as $(x, y) \in G_{1}(\omega)$ (a.s.), which follows from (33). The second inequality is a consequence of (37) and the third one holds by virtue of (39). The last equality is valid since

$$
\frac{\tilde{p}_{2}}{\tilde{\lambda}_{1}}=\frac{\left|x_{1}\right| a_{2}}{\lambda_{1}\left|x_{1}\right| /\left|x_{0}\right|}=\frac{\left|x_{0}\right| a_{2}}{\lambda_{1}}
$$

From (40) we obtain (in view of the equivalence of (2) and (3) that with probability one

$$
\frac{E_{1} \tilde{p}_{1}(T \omega) b}{\lambda_{1}(\omega)} \leq p(\omega) a \text { for all }(a, b) \in G_{1}(\omega)
$$

We have

$$
\tilde{p}_{1} \tilde{x}_{0}=\left|x_{0}\right| a_{1} \frac{x_{0}}{\left|x_{0}\right|}=a_{1} F_{0} u_{0} \leq q_{1} u_{0}=1 \text { (a.s.) }
$$

where the inequality follows from (38). Further, we get

$$
E \tilde{p}_{1} \tilde{x}_{0}=E a_{1} x_{0} \geq E b_{1} \lambda_{1} x_{1}=E b_{1} F_{1}\left(\lambda_{1} u_{1}\right) \geq E \frac{\bar{q}_{2}}{\lambda_{1}} \lambda_{1} u_{1}=E q_{2} u_{1}=1 .
$$

In this chain of relations, the first inequality holds by virtue of (33) and because $\left(x_{0}, \lambda_{1} x_{1}\right) \in G_{1}(\omega)$ a.s. (see (36)). The second follows from (37). By combining (41) and (42), we conclude that $\tilde{p}_{1} \tilde{x}_{0}=1$ (a.s.). 
Let us show that for almost all $\omega \in \Omega$,

$$
\tilde{p}_{1}(\omega) a \geq 0 \text { for } a \in X_{0}(\omega)
$$

i.e. $\tilde{p}_{1}(\omega) \in X_{0}^{*}(\omega)$ (a.s.). Denote by $\Omega^{1}$ the set of those $\omega$ for which $b_{1}(\omega) F_{1}(\omega) \geq 0$. By virtue of (37), we have $P\left(\Omega^{1}\right)=1$. Fix any $\omega \in \Omega^{1}, a \in X_{0}(\omega)$ and consider some $c \in \mathbb{R}_{+}^{K}$ for which $F_{0}(\omega) c=a$. By virtue of $\left(\mathbf{H} \mathbf{1}^{\prime}\right)$, there exists $d \in \mathbb{R}_{+}^{K}$ such that $(c, d) \in H_{1}(\omega)$. Then $b_{1} F_{1} d \leq a_{1} F_{0} c$ in view of (33). From this we get

$$
\tilde{p}_{1} a=\left|x_{0}\right| a_{1} F_{0} c \geq\left|x_{0}\right| b_{1} F_{1} d \geq 0 .
$$

It remains to show that the balanced path generated by $\left(\tilde{x}_{0}, \tilde{\lambda}_{1}\right)$ is a von Neumann path, i.e. $E \ln \lambda_{1}^{\prime} \leq E \ln \tilde{\lambda}_{1}$ as long as $\lambda_{1}^{\prime}$ is an $\mathcal{F}_{1}$-measurable scalar function with $E\left|\ln \lambda_{1}^{\prime}\right|<+\infty$ and such that $\left(x_{0}^{\prime}, \lambda_{1}^{\prime} T x_{0}^{\prime}\right) \in G_{1}(\omega)$ (a.s.) for some $x_{0}^{\prime} \in \mathcal{X}_{0}$ with $\left|x_{0}^{\prime}(\omega)\right|=1$. (a.s.). Consider an $\mathcal{F}_{0}$-measurable function $u_{0}^{\prime}(\omega)$ such that $F_{0} u_{0}^{\prime}=x_{0}^{\prime}$. By virtue of $(8), \Theta^{-1} \leq\left|u_{0}^{\prime}\right| \leq \theta^{-1}$. We have

$$
F_{1}\left(\lambda_{1}^{\prime} T u_{0}^{\prime}\right)=\lambda_{1}^{\prime} F_{1}\left(T u_{0}^{\prime}\right)=\lambda_{1}^{\prime} T F_{0}\left(u_{0}^{\prime}\right)=\lambda_{1}^{\prime} T x_{0}^{\prime} .
$$

Since $\left(x_{0}^{\prime}, \lambda_{1}^{\prime} T x_{0}^{\prime}\right) \in G_{1}(\omega)$ (a.s.), $\left(u_{0}^{\prime}, \lambda_{1}^{\prime} T u_{0}^{\prime}\right) \in H_{1}(\omega)$ (a.s.), and consequently

$$
\left(\frac{u_{0}^{\prime}}{\left|u_{0}^{\prime}\right|}, \frac{\lambda_{1}^{\prime}\left|T u_{0}^{\prime}\right|}{\left|u_{0}^{\prime}\right|} \frac{T u_{0}^{\prime}}{\left|T u_{0}^{\prime}\right|}\right) \in H_{1}(\omega) .
$$

By using (33), we get

$$
E \ln \lambda_{0}^{\prime}=E \ln \frac{\lambda_{1}^{\prime}\left|T u_{0}^{\prime}\right|}{\left|u_{0}^{\prime}\right|} \leq E \ln \lambda_{1}=E \ln \tilde{\lambda}_{1},
$$

which shows that the triplet $\left(\tilde{x}_{0}, \tilde{p}_{1}, \tilde{\lambda}_{1}\right)$ forms a von Neumann equilibrium in the dynamical system $\mathcal{G}$.

An existence theorem for a von Neumann equilibrium in a canonical stationary von Neumann-Gale dynamical system was obtained in [24], Theorem 1 and Proposition 3. To formulate this result we introduce the following additional assumption (known in Mathematical Economics as the "free disposal hypothesis"):

(FD) If $(c, d) \in H_{1}(\omega), c^{\prime} \geq c$ and $0 \leq d^{\prime} \leq d$, then $\left(c^{\prime}, d^{\prime}\right) \in H_{1}(\omega)$.

Theorem 5.3. Under assumptions $\left(\boldsymbol{H} \mathbf{1}^{\prime}\right),\left(\boldsymbol{H}^{\prime}\right),\left(\boldsymbol{h} \boldsymbol{4}^{\prime}\right)$ and $(\boldsymbol{F D})$, a von Neumann equilibrium exists.

It can be shown that condition (FD) in the present context is redundant: Theorem 5.3 holds without it. To this end, let us define

$$
\hat{H}_{1}(\omega):=\left\{(c, d) \in \mathbb{R}_{+}^{K} \times \mathbb{R}_{+}^{K}: \text { there exists } \hat{d} \geq d \text { such that }(c, \hat{d}) \in H_{1}(\omega)\right\}
$$

It is clear that $\hat{H}_{1}(\omega)$ is a cone and $H_{1}(\omega) \subseteq \hat{H}_{1}(\omega)$.

Proposition 5.4. $\hat{H}_{1}(\omega)$ satisfies (FD). 
Proof. Let $(c, d) \in \hat{H}_{1}(\omega)$ and $0 \leq d^{\prime} \leq d$. Since $(c, d) \in \hat{H}_{1}(\omega)$, there exists $\hat{d} \geq d \geq 0$ such that $(c, \hat{d}) \in H_{1}(\omega)$. Then $\left(c, d^{\prime}\right) \in \hat{H}_{1}(\omega)$, because $\hat{d} \geq d \geq d^{\prime} \geq 0$. Observe that if $(c, d) \in \hat{H}_{1}(\omega)$, then $(c, 0) \in \hat{H}_{1}(\omega)$.

Let $(c, d) \in \hat{H}_{1}(\omega)$ and $c^{\prime} \geq c$. Put $c^{\prime}=c+h, h \geq 0$. By virtue of condition $\left(\mathbf{H 1}^{\prime}\right),(h, g) \in H_{1}(\omega) \subseteq \hat{H}_{1}(\omega)$ for some $g \geq 0$, then $(h, 0) \in \hat{H}_{1}(\omega)$. Therefore $\left(c^{\prime}, d\right)=(c+h, d) \in \hat{H}_{1}(\omega)$.

Proposition 5.5. If $(u, q, \lambda)$ is a von Neumann equilibrium for $\hat{H}_{1}(\omega)$, then it is a von Neumann equilibrium for $H_{1}(\omega)$.

Proof. By definition of von Neumann equilibrium, $u(\omega) \geq 0$ is an $\mathcal{F}_{0}$-measurable vector function normalized by the condition $|u(\omega)|=1$ (a.s.), $\lambda(\omega)>0$ is an $\mathcal{F}_{1}$ measurable scalar function with $E \ln \lambda(\omega)<+\infty, q(\omega) \in L_{t}^{1,+}\left(\mathbb{R}^{K}\right)$ such that

$$
(u(\omega), \lambda(\omega) u(T \omega)) \in \hat{H}_{1}(\omega)(\text { a.s. })
$$

for almost all $\omega$

$$
\frac{E_{1} q(T \omega) b}{\lambda(\omega)} \leq q(\omega) a \text { for all }(a, b) \in \hat{H}_{1}(\omega)
$$

and $q(\omega) u(\omega)=1$ (a.s.).

By virtue of (43) and the measurable selection theorem, there exists an $\mathcal{F}_{1-}$ measurable vector function $g(\omega) \geq 0$ such that $(u(\omega), \lambda(\omega) u(T \omega)+g(\omega)) \in H_{1}(\omega)$ (a.s.). By applying (44) to $(u(\omega), \lambda(\omega) u(T \omega)+g(\omega))$ and using the fact that $q(\omega) u(\omega)=1$ (a.s.), we obtain

$$
E q(T \omega) g(\omega) \leq 0
$$

Since $q(T \omega) g(\omega) \geq 0$ (a.s.), we conclude that $q(T \omega) g(\omega)=0$ (a.s.) and so $g(\omega)=$ 0 (a.s.) because $q(T \omega) \neq 0$ (a.s.). Therefore $(u(\omega), \lambda(\omega) u(T \omega)) \in H_{1}(\omega)$ (a.s.) and $(u, q, \lambda)>0$ is a von Neumann equilibrium for $H_{1}(\omega)$. The proof is complete.

Proof of Theorem 3.5. By virtue of Proposition 5.1, conditions $\left(\mathbf{H} 1^{\prime}\right),\left(\mathbf{H 2}^{\prime}\right)$ and $\left(\mathbf{H} 4^{\prime}\right)$ hold. Note that these conditions hold true for $\hat{H}_{1}(\omega)$. Therefore from Theorem 5.3 and Proposition 5.4, we conclude that there exists a triplet of functions $(u, q, \lambda)$ forming a von Neumann equilibrium for $\hat{H}_{1}(\omega)$ which is also an equilibrium for $H_{1}(\omega)$ by virtue of Proposition 5.5. Consequently, Theorem 5.2 guarantees that there exists a triplet of functions $(x, p, \lambda)$ forming a von Neumann equilibrium in the dynamical system $\mathcal{G}$.

\section{A multicurrency model for a market with dividend-paying assets}

In this section we describe a specialized model for a financial market with frictions and provide conditions guaranteeing that Theorems 3.2, 3.4, and 3.5 can be applied to this model. We consider a market where $N$ assets are traded at dates $t=1,2, \ldots$ A portfolio of assets is represented by a vector $a=\left(a^{1}, \ldots, a^{N}\right) \in \mathbb{R}^{N}$. The $i$ th component 
of this vector is equal to the value of the portfolio position corresponding to asset $i$. The value is measured in terms of some numéraire. It is typically assumed that the numéraire is the base currency (domestic cash). It does not necessarily need to be one of the $N$ assets. The set $\{1,2, \ldots, N\}$ of all the assets is decomposed into two subsets, $I_{1}$ and $I_{2}$, each of which may be empty. Those assets that are indexed by $i \in I_{1}$ represent currencies $^{3}$ and those labeled by $i \in I_{2}$ represent assets of other kind, typically shares of stock.

For each $t \geq 1$ and $i, j=1, \ldots, N$, the following $\mathcal{F}_{t}$-measurable random variables are given: the asset prices $q_{t, i}>0$ quoted in units of the numéraire, transaction cost rates $0 \leq \lambda_{t, i, j}<1(i \neq j)$ for exchanging asset $i$ for asset $j$, and dividend yields or interest rates $0 \leq D_{t, i, j}^{+} \leq D_{t, i, j}^{-}$for long and short positions. If $a=\left(a^{1}, \ldots, a^{N}\right) \in \mathbb{R}^{N}$ is a portfolio, then $a^{i} / q_{t, i}$ is the number of units of asset $i$ held in it and $R_{t, i}=q_{t, i} / q_{t-1, i}$ is the (gross) return on asset $i$.

The variables $\lambda_{t, i, j}$ have the following meaning. Suppose the trader reduces the value of her $i$ th portfolio position $a^{i}$ by $\theta \geq 0$ units of numéraire with the view to increasing the $j$ th position $a^{j}$. Then the amount added to $a^{j}$ will be equal to $\theta-\lambda_{t, i, j} \theta$, where $\lambda_{t, i, j} \theta$ is the transaction cost rate. This operation comprises currency exchange when $i, j \in I_{1}$, buying and selling assets $j \in I_{2}$ for currencies $i \in I_{1}$, and barter trading when $i, j \in J_{2}$.

The meaning of the variables $D_{t, i, j}^{ \pm}$is as follows. If $i \in I_{1}$, i.e. $i$ represents a currency, then $D_{t, i, j}^{+}$and $D_{t, i, j}^{-}$might be non-zero only if $j=i$ : the interest is paid in the same currency (but measured in terms of the numeraire). If $a^{i}>0$, then $D_{t, i, i}^{+} a^{i}$ is the interest paid by the amount of currency $i$ worth $a^{i}>0$ units of numéraire. When $a^{i}<0$, i.e. some amount of currency $i$ has been borrowed, say, from a bank, then $D_{t, i, i}^{-} i^{i}$ indicates the amount that has to be returned, so that $D_{t, i, i}^{-}$represents the bank's interest rate for lending. The value of the dividends (in units of the numeraire) that is paid by asset $i \in I_{2}$ and added to the position corresponding to asset $j \in I_{1}$ is equal to $D_{t, i, j}^{+} a_{+}^{i}-D_{t, i, j}^{-} a_{-}^{i}$. It is natural to assume that the dividends on stock $i \in I_{2}$ are paid in some currency $j(i) \in I_{1}$, so that $D_{t, i, j}^{ \pm}=0$ for $j \neq j(i)$.

The market in the model at hand is organized as follows. At each date $t$, the trader receives dividends and interest $D_{t, i, j}^{+} a_{+}^{i}-D_{t, i, j}^{-} a_{-}^{i}$ on her portfolio $a(\omega)$ purchased at the previous date $t-1$. These amounts (positive or negative) are added to the corresponding portfolio positions. After receiving dividend and interest payments, the trader will have the portfolio $d_{t}(\omega, a)=\left(d_{t}^{1}(\omega, a), \ldots, d_{t}^{N}(\omega, a)\right)$ with the positions

$$
d_{t}^{j}(a)=R_{t, j} a^{j}+\sum_{i=1}^{N}\left(D_{t, i, j}^{+} a_{+}^{i}-D_{t, i, j}^{-} a_{-}^{i}\right), j=1, \ldots, N,
$$

where the first term in the right-hand side represents the new value (in units of the numéraire) of the portfolio position in asset $j$ after the change of its price. Then the trader can rearrange her portfolio into a new portfolio $b(\omega)$. The model assumes that such a rearrangement is possible if and only if there exists an $N \times N$ matrix $\Theta=\left(\theta_{i, j}\right)$

\footnotetext{
${ }^{3}$ Models of currency markets with proportional transaction costs (bid-ask spreads) were developed by Kabanov and co-authors-see, e.g., [39, 42] and references therein.
} 
(a matrix of transactions) with non-negative elements such that for each $j=1, \ldots, N$

$$
d_{t}^{j}(a)+\sum_{i=1}^{N}\left(\left(1-\lambda_{t, i, j}\right) \theta_{i, j}-\theta_{j, i}\right) \geq b^{j} .
$$

The element $\theta_{i, j}$ of the matrix $\Theta$ is the value of the part of the position in asset $i$ which is exchanged for asset $j$, and $\lambda_{t, i, j} \theta_{i, j}$ is the corresponding transaction cost.

Denote the left-hand side of (45) by $\psi_{t}^{j}(\omega, a, \Theta)$ and consider the vector-valued function $\psi_{t}(\omega, a, \Theta)$ with the components $\psi_{t}^{j}(\omega, a, \Theta), j=1, \ldots, N$. The above description of the model corresponds to the cones

$$
G_{t}(\omega):=\left\{(a, b) \in X_{t-1}(\omega) \times X_{t}(\omega): \exists \Theta \in \mathbb{R}_{+}^{N \times N}: \psi_{t}(\omega, a, \Theta) \geq b\right\}
$$

where the inequality should be understood coordinatewise. As long as $X_{t-1}$ and $X_{t}$ are cones, the fact that $G_{t}$ are cones follows from (45), where we need the assumption $D_{t, i, j}^{+} \leq D_{t, i, j}^{-}$to ensure that the functions $d_{t}^{j}(a)$ are concave.

We will consider the cones $X_{t}$, that define portfolio constraints, of the following form:

$$
X_{t}(\omega)=\left\{a \in \mathbb{R}^{N}: \sum_{i=1}^{N} \mu_{t, i}^{+}(\omega) a_{+}^{i} \geq \sum_{i=1}^{N} \mu_{t, i}^{-}(\omega) a_{-}^{i}\right\}
$$

where $0<\mu_{t, i}^{+} \leq \mu_{t, i}^{-}$are positive $\mathcal{F}_{t}$-measurable random variables. These random variables can be used to specify margin requirements, as, for example, in the following two particular models:

$$
X_{t}(\omega)=\left\{a \in \mathbb{R}^{N}:\left|a_{+}\right| \geq U_{t}\left|a_{-}\right|\right\},
$$

or

$$
X_{t}(\omega)=\left\{a \in \mathbb{R}^{N}: a_{+}^{1}+\sum_{i=2}^{N}\left(1-\lambda_{t, i, 1}\right) a_{+}^{i} \geq U_{t}\left(a_{-}^{1}+\sum_{i=2}^{N}\left(1-\lambda_{t, 1, i}\right)^{-1} a_{-}^{i}\right)\right\},
$$

where $U_{t}>1$ are constants interpreted as margin coefficients: the trader must be able to cover the short positions of the portfolio by its long positions with excess determined by $U_{t}$. In (46) no transaction costs are taken into account in the computation of the values of the short and long positions. In (47), the transaction costs are calculated assuming that all the transactions performed to cover the short positions are done through asset 1 (the base currency).

Note that the expression in parentheses on the right-hand side of the inequality in (47) is equal to the numéraire value of the amount of asset 1 that is needed to close all the short positions under transaction costs. The amount of asset 1 that is worth one unit of the numeraire (i.e. $1 / q_{t, 1}$ units of asset 1 ) can be exchanged for the amount of asset $i$, which has the value of $\left(1-\lambda_{t, 1, i}\right)^{-1}$ units of the numeraire. Similarly, the left-hand side of the inequality in (47) is the value of the amount of asset 1 which can be obtained by exchanging all the long positions for asset 1 . 
As a liquidation value function, which appears in condition $(\mathbf{L})$, we will use

$$
\phi_{t}(\omega, b)=b^{1}+\sum_{i=2}^{N}\left(1-\lambda_{t, i, 1}(\omega)\right) b_{+}^{i}-\sum_{i=2}^{N}\left(1-\lambda_{t, 1, i}(\omega)\right)^{-1} b_{-}^{i} .
$$

This function is equal to the numéraire value of the position in asset 1 that the trader can obtain if she closes all the other positions, assuming that all the transactions are done through asset 1, i.e. the long positions in assets $i=2, \ldots, N$ are exchanged for asset 1 , and then asset 1 is exchanged to close all the short positions in assets $i=2, \ldots, N$.

Now we provide sufficient conditions to guarantee that this model satisfies conditions (F), (G1)-(G4), (L), and so Theorems 3.2 and 3.4 can be applied to it. Let $\Lambda_{t, i}^{+}=$ $1-\lambda_{t, i, 1}, \Lambda_{t, i}^{-}=\left(1-\lambda_{t, 1, i}\right)^{-1}$ for $i=2, \ldots, N$, and $\Lambda_{t, 1}^{ \pm}=1$.

We introduce the following conditions.

(A1) For each $t$, there exist constants $\underline{R}_{t}, \bar{R}_{t}, \underline{\Lambda}_{t}, \bar{\Lambda}_{t}$, and $\bar{D}_{t}$ such that $0<\underline{R}_{t} \leq$ $R_{t, i}(\omega) \leq \bar{R}_{t}, D_{t, i, j}^{-}(\omega) \leq \bar{D}_{t}, 0<\underline{\Lambda}_{t} \leq \Lambda_{t, i}^{+}(\omega)$, and $\Lambda_{t, i}^{-}(\omega) \leq \bar{\Lambda}_{t}$ for all $i, j, \omega$.

(A2) For each $t$, there exists a constant $\mu_{t}$ such that $\mu_{t, i}^{-}(\omega) / \mu_{t, j}^{+}(\omega) \geq \mu_{t}$ for all $\omega$, $i \neq j$, and $\mu_{t}>\nu_{t}$, where

$$
\nu_{t}:=\left(1+\frac{\bar{\Lambda}_{t+1}}{\underline{\Lambda}_{t+1}}\right) \frac{\bar{R}_{t+1}+N \bar{D}_{t+1}}{\underline{R}_{t+1}+N \underline{D}_{t+1}}
$$

and $\underline{D}_{t} \geq 0$ is a constant such that $\underline{D}_{t} \leq D_{t, i, j}^{+}(\omega)$ for all $\omega, i, j$.

The financial meaning of conditions (A1) and (A2) is as follows. The first two inequalities in (A1) require the ratio $R_{t}$ of asset prices at two consecutive dates to be bounded away from zero and bounded from above by some constants. The third inequality says that the dividend yields cannot be greater than the constants $\bar{D}_{t}$. The last inequalities state that the transaction costs both for buying and selling should not be "too high". Condition (A2) requires that the size of the margin determined by the relation $\mu_{t, i}^{-}(\omega) / \mu_{t, j}^{+}(\omega)$ should not be "too small" — at least, greater than $\nu_{t}$. These conditions are needed to verify the validity of assumptions $(\mathbf{G 1})-(\mathbf{G} \mathbf{4})$ and $(\mathbf{F})$.

Observe that for the particular example of the cones $X_{t}(\omega)$ in (46), if condition (A1) is satisfied, then (A2) will hold if $U_{t}>\nu_{t}$ for each $t$. In (47), (A2) will hold if (A1) holds and $U_{t} \geq \nu_{t} \Lambda_{t, i}^{+} / \Lambda_{t, j}^{-}+\epsilon_{t}$ for each $t$ and $i \neq j$, where $\epsilon_{t}>0$ are some constants.

Proposition 6.1. Let conditions (A1), (A2) hold. Then:

(a) the cones $X_{t}(\omega)$ satisfy condition $(\boldsymbol{F})$ and the cones $G_{t}(\omega)$ satisfy conditions (G1)(G3);

(b) if $\mu_{t}, \underline{R}_{t}, \bar{R}_{t}, \underline{\Lambda}_{t}, \bar{\Lambda}_{t}, \bar{D}_{t}$ do not depend on $t$, then $G_{t}(\omega)$ satisfy condition (G2) with constant $M$ not depending on $t$ and condition (G4) with $m=1$; if additionally and $\mu \underline{\Lambda}>\bar{\Lambda}$, then the function $\phi_{t}$ defined in (48) satisfies condition $(\boldsymbol{L})$.

We will need the following auxiliary result to prove Proposition 6.1.

Lemma 6.2. Let conditions (A1), (A2) hold. Then

(a) For each $t$ there exists a constant $C_{t}^{1}>0$ such that if $a \in X_{t}(\omega)$ then $\left|a_{+}\right|-$ $\nu_{t}\left|a_{-}\right| \geq C_{t}^{1}|a|$.

(b) For each $t$ there exists a constant $C_{t}^{2}$ such that if $a \in X_{t-1}(\omega), b \in X_{t}(\omega)$ and 
$|b| \leq C_{t}^{2}|a|$, then $(a, b) \in G_{t}(\omega)$.

Proof. (a) Consider the non-random cone $\tilde{X}_{t}=\left\{a \in \mathbb{R}^{N}: \mu_{t}\left|a_{-}\right| \leq\left|a_{+}\right|\right\}$. Condition (A2) implies that $X_{t}(\omega) \subseteq \tilde{X}_{t}$, i.e. $X_{t}(\omega)$ satisfies condition $(\mathbf{M})$ with constant $\mu_{t}$. Observe that the continuous function $h_{t}(a)=\left|a_{+}\right|-\nu_{t}\left|a_{-}\right|$is strictly positive on the compact set $K_{t}=\tilde{X}_{t} \cap\{a:|a|=1\}$. Indeed, since $h_{t}(a) \geq\left(\mu_{t}-\nu_{t}\right)\left|a_{-}\right|$on $\tilde{X}_{t}$, then the equality $h_{t}(a)=0$ would imply $\left|a_{-}\right|=0$, and hence $\left|a_{+}\right|=h_{t}(a)=0$, so that $|a|=0$. Then $h_{t}(a)$ attains a strictly positive minimum on $K_{t}$, which can be taken as $C_{t}^{1}$.

(b) Let $a \in X_{t-1}(\omega), b \in X_{t}(\omega)$. Consider the transaction matrix $\Theta$ with the elements $\theta_{i, 1}=\left(d_{t}^{i}(a)-b^{i}\right)_{+}, \theta_{1, i}=\left(1-\lambda_{t, 1, i}\right)^{-1}\left(d_{t}^{i}(a)-b^{i}\right)_{-}$for $i=2, \ldots, N$ and all the other elements being zero. Then $\psi_{t}^{i}(a, \Theta)=b^{i}$ for $i=2, \ldots, N$.

It is straightforward to check that for any numbers $x, y$ we have $(x-y)_{+} \geq x_{+}-y_{+}$ and $(x-y)_{-} \leq x_{-}+y_{+}$. Using this, we obtain

$$
\begin{aligned}
& \psi_{t}^{1}(a, \Theta)-b^{1}=\sum_{i=1}^{N}\left(\Lambda_{t, i}^{+}\left(d_{t}^{i}(a)-b^{i}\right)_{+}-\Lambda_{t, i}^{-}\left(d_{t}^{i}(a)-b^{i}\right)_{-}\right) \\
& \geq \sum_{i=1}^{N}\left(\Lambda_{t, i}^{+}\left[d_{t}^{i}(a)\right]_{+}-\Lambda_{t, i}^{-}\left[d_{t}^{i}(a)\right]_{-}-\left(\Lambda_{t, i}^{+}+\Lambda_{t, i}^{-}\right) b_{+}^{i}\right) \\
& \geq \sum_{i=1}^{N}\left(\underline{\Lambda}_{t}\left[d_{t}^{i}(a)\right]_{+}-\bar{\Lambda}_{t}\left[d_{t}^{i}(a)\right]_{-}\right)-\left(1+\bar{\Lambda}_{t}\right)|b| .
\end{aligned}
$$

Observe that

$$
\begin{gathered}
{\left[d_{t}^{i}(a)\right]_{+} \geq d_{t}^{i}(a) \geq \underline{R}_{t} a_{+}^{i}+\underline{D}_{t}\left|a_{+}\right|-\bar{R}_{t} a_{-}^{i}-\bar{D}_{t}\left|a_{-}\right|} \\
{\left[d_{t}^{i}(a)\right]_{-} \leq \bar{R}_{t} a_{-}^{i}+\bar{D}_{t}\left|a_{-}\right| .}
\end{gathered}
$$

Hence

$$
\begin{aligned}
\psi_{t}^{1}(a, \Theta)-b^{1} & \geq \underline{\Lambda}_{t}\left(\underline{R}_{t}+N \underline{D}_{t}\right)\left|a_{+}\right|-\left(\underline{\Lambda}_{t}+\bar{\Lambda}_{t}\right)\left(\bar{R}_{t}+N \bar{D}_{t}\right)\left|a_{-}\right|-\left(1+\bar{\Lambda}_{t}\right)|b| \\
& =\underline{\Lambda}_{t}\left(\underline{R}_{t}+N \underline{D}_{t}\right)\left(\left|a_{+}\right|-\nu_{t-1}\left|a_{-}\right|\right)-\left(1+\bar{\Lambda}_{t}\right)|b| \\
& \geq C_{t-1}^{1} \underline{\Lambda}_{t}\left(\underline{R}_{t}+N \underline{D}_{t}\right)|a|-\left(1+\bar{\Lambda}_{t}\right)|b| .
\end{aligned}
$$

Then statement (b) can be fulfilled with the constant $C_{t}^{2}=C_{t-1}^{1} \underline{\Lambda}_{t}\left(\underline{R}_{t}+N \underline{D}_{t}\right) /\left(1+\bar{\Lambda}_{t}\right)$, since in that case $\psi_{t}^{1}(a, T)-b^{1} \geq 0$, implying $(a, b) \in G_{t}$.

Proof of Proposition 6.1. (a) Let us show that each cone $X_{t}$ is polyhedral. Put $f_{t, i, j}=e_{i}-\left(\mu_{t, i}^{+} / \mu_{t, j}^{-}\right) e_{j}$ for $i \neq j$, where $e_{i}$ is the $i$-th basis vector in $\mathbb{R}^{N}$. Suppose $a \in X_{t}(\omega), a \neq 0$. Denote by $I=\left\{i: a^{i}>0\right\}, J=\left\{j: a^{j}<0\right\}$ the sets of indices of positive and negative coordinates of $a$ and put $\delta=\left(\sum_{j \in J} \mu_{t, j}^{-}\left|a^{j}\right|\right) /\left(\sum_{i \in I} \mu_{t, i}^{+} a^{i}\right)$. Clearly, $\delta \leq 1$ as $a \in X_{t}$. Then

$$
a=\delta \sum_{i \in I} \sum_{j \in J} \frac{a^{i} \mu_{t, j}^{-}\left|a^{j}\right|}{\sum_{k \in J} \mu_{t, k}^{-}\left|a^{k}\right|} f_{t, i, j}+(1-\delta) \sum_{i \in I} a^{i} e_{i} .
$$

Hence the cone $X_{t}$ can be represented in the form (5) with $N^{2}$ generators: $f_{t, i, j}$ and $e_{i}$ 
for $i, j=1, \ldots, N, j \neq i$. As it was noted in the proof of statement (a) of Lemma 6.2, the cones $X_{t}$ satisfy $(\mathbf{M})$. So, by Proposition 3.1, they also satisfy $(\mathbf{F})$.

Condition (G1) follows from that, according to statement (b) of Lemma 6.2, for any $a \in X_{t-1}(\omega)$ we have $(a, 0) \in G_{t}(\omega)$.

Let us prove (G2). Suppose $(a, b) \in G_{t}$. Since $b \in X_{t}(\omega)$, statement (a) of Lemma 6.2 implies that $|b| \leq\left(C_{t}^{1}\right)^{-1} \sum_{i=1}^{N} b^{i}$. Moreover, there exists a transaction matrix $\Theta$ for which (45) holds, so we have

$$
|b| \leq \frac{1}{C_{t}^{1}} \sum_{i=1}^{N} d_{t}^{i}(a) \leq \frac{1}{C_{t}^{1}}\left(\bar{R}_{t}+N \bar{D}_{t}\right)|a| .
$$

This implies the validity of $(\mathbf{G 2})$ with constant $M_{t}=\left(\bar{R}_{t}+N \bar{D}_{t}\right) / C_{t}^{1}$.

Now we will prove condition $(\mathbf{G 3})$. Let $\hat{x}=(1, \ldots, 1) \in \mathbb{R}^{N}$. Put $\hat{z}_{t}=\left(\hat{x}, \hat{y}_{t}\right)$ with $\hat{y}_{t}=\left(C_{t}^{2} / 2\right) \hat{x}$. Observe that there exists $\delta_{t}>0$ such that $\mathbb{B}\left(\hat{z}_{t}, \delta_{t}\right) \subset \mathbb{R}_{+}^{2 N}$ and therefore $\mathbb{B}\left(\hat{z}_{t}, \delta_{t}\right) \subset X_{t-1} \times X_{t}$. Since $\left|\hat{y}_{t}\right|<C_{t}^{2}|\hat{x}|$, then one can find $0<\alpha_{t} \leq \delta_{t}$ such that $\left|y_{t}\right| \leq C_{t}^{2}\left|x_{t}\right|$ for any $z_{t}=\left(x_{t-1}, y_{t}\right) \in \mathbb{B}\left(\hat{z}_{t}, \alpha_{t}\right)$. Then statement (b) of Lemma 6.2 implies $z_{t} \in G_{t}$ for such $z_{t}$. Hence, the pair $\left(\hat{z}_{t}, \alpha_{t}\right)$ satisfies condition (G3).

(b) From the proof of statement (a) of Lemma 6.2 one can see, that if the constants from condition (A1) do not depend on $t$, then it is possible to choose $C_{t}^{1}$ independent of $t$. Then (49) implies that $M_{t}$ can be chosen independent of $t$.

Let us prove that $(\mathbf{G 4})$ holds. It follows from the proof of Lemma 6.2, that the constant $C_{t}^{2}$ can be chosen independent of $t$. Let $\gamma=C^{2} /(N+1)$ and consider any $y_{t} \in \mathcal{X}_{t}$. Put $y_{t+1}=\gamma\left|y_{t}\right| \hat{x}$. Then $\mathbb{B}\left(y_{t+1}, \gamma\left|y_{t}\right|\right) \subseteq \mathbb{R}_{+}^{N} \subseteq X_{t+1}$. Hence for any $y \in \mathcal{X}_{t+1}$ such that $|y| \leq \gamma\left|y_{t}\right|$ we have $y_{t+1}+y \in X_{t+1}$, and then statement (b) of Lemma 6.2 implies that $\left(y_{t}, y_{t+1}+y\right) \in G_{t+1}$, so condition (G4) holds with $m=1$.

Finally, we prove that the function $\phi_{t}(\omega, b)$ satisfies condition $(\mathbf{L})$. Observe that it can be represented in the form $\phi_{t}(b)=\sum_{i=1}^{N}\left(\Lambda_{t, i}^{+} b_{+}^{i}-\Lambda_{t, i}^{-} b_{-}^{i}\right)$. From the proof of Lemma 6.2 , it follows that the cones $X_{t}$ satisfy condition (M) with constant $\mu$ independent of $t$. Using the condition $\mu \underline{\Lambda}>\bar{\Lambda}$ and applying Proposition 3.3, we see that $(\mathbf{L})$ is satisfied.

Now we provide a sufficient condition for the existence of a von Neumann equilibrium in the stationary version of the model.

Proposition 6.3. Let $T$ be an automorphism of the underlying probability space such that

$$
\begin{gathered}
\mathcal{F}_{t+1}=T^{-1}\left(\mathcal{F}_{t}\right), \mu_{t+1, i}^{ \pm}(\omega)=\mu_{t, i}^{ \pm}(T \omega), R_{t+1, i}(\omega)=R_{t, i}(T \omega), . \\
\lambda_{t+1, i, j}(\omega)=\lambda_{t, i, j}(T \omega), D_{t+1, i, j}^{ \pm}(\omega)=D_{t, i, j}^{ \pm}(T \omega) .
\end{gathered}
$$

Let conditions (A1) and (A2) hold for some $t$ and hence for all $t$. Then a von Neumann equilibrium exists.

The validity of this proposition follows from Proposition 6.1 and Theorem 3.5. 


\section{Appendix}

\subsection{A measurable selection theorem}

Let $(\Omega, \mathcal{F}, P)$ be a probability space such that the $\sigma$-algebra $\mathcal{F}$ is complete with respect to measure $P$ (all subsets of $\mathcal{F}$-measurable sets of measure 0 are $\mathcal{F}$-measurable). Let $B$ be a complete separable metric space and $\mathcal{B}$ its Borel $\sigma$-algebra. Let $\omega \mapsto A(\omega)$ be a multivalued mapping assigning a non-empty set $A(\omega) \subseteq B$ to each $\omega \in \Omega$.

Theorem 7.1. If $\{(\omega, a): a \in A(\omega)\} \in \mathcal{F} \times \mathcal{B}$, then for each $\omega$ one can select a point $\alpha(\omega) \in A(\omega)$ such that the mapping $\alpha:(\Omega, \mathcal{F}) \rightarrow(B, \mathcal{B})$ is measurable.

For a proof of this result see, e.g., Castaing and Valadier [11].

\subsection{The Kuhn-Tucker theorem}

We formulate a version of this theorem that is used in this work. Let $X \subset \mathbb{R}^{n}$ be a convex set, $\Phi: X \rightarrow \mathbb{R}^{1}$ a concave function, $R: \mathbb{R}^{n} \rightarrow \mathbb{R}^{m}$ a linear mapping and $Z$ a cone in $\mathbb{R}^{m}$. Assume that the following assumption (Slater's condition) holds.

(S) There exists an element $\hat{x}$ of the set $X$ such that the point $\hat{z}:=R \hat{x}$ is contained in $Z$ together with a ball $\mathbb{B}(\hat{z}, \gamma)(\gamma>0)$.

Theorem 7.2. Let $x^{*}$ be a point in $X$ where the function $\Phi(x)$ attains its maximum on $X$ subject to the constraint $R x \in Z$. Then there exists a linear functional $g$ on $\mathbb{R}^{m}$ such that

$$
\Phi(x)-g R x \leq \Phi\left(x^{*}\right), x \in X,
$$

and

$$
g z \leq 0, z \in Z
$$

Furthermore, we have

$$
|g| \leq m \gamma^{-1}\left[\Phi\left(x^{*}\right)-\Phi(\hat{x})\right] .
$$

Proof. For the existence of $g$ see, e.g., Luenberger [33]. The estimate (52) is obtained as follows. We have

$$
0 \geq g \hat{z}=g R \hat{x} \geq \Phi(\hat{x})-\Phi\left(x^{*}\right),
$$

where the first inequality follows from (51) and the second from (50). Since $\mathbb{B}(\hat{z}, \gamma) \subseteq Z$, if $|h| \leq \gamma$, then $\hat{z}-h \in Z$, and so $g(\hat{z}-h) \leq 0$, i.e. $g h \geq g \hat{z}$. By combining this inequality with (53), we get

$$
g h \geq g \hat{z} \geq \Phi(\hat{x})-\Phi\left(x^{*}\right)
$$

for any $h$ such that $|h| \leq \gamma$. Therefore

$$
|g h| \leq \Phi\left(x^{*}\right)-\Phi(\hat{x})
$$


for all $h$ with $|h| \leq \gamma$. Put $h=\gamma m^{-1 / 2}\|g\|^{-1} g$, where $\|\cdot\|$ is the Euclidean norm in $\mathbb{R}^{m}$. Then $|h|=\gamma m^{-1 / 2}|| g \|^{-1}|g| \leq \gamma$ because $|g| \leq \sqrt{m}\|g\|$ for each $g \in \mathbb{R}^{m}$. From (54) we obtain

$$
\Phi\left(x^{*}\right)-\Phi(\hat{x}) \geq|g h|=\gamma m^{-1 / 2}|| g \|\left.^{-1}|| g\right|^{2}=\gamma m^{-1 / 2}|| g|| \geq \gamma m^{-1}|g|,
$$

which proves (52).

\section{Funding}

Mikhail Zhitlukhin's research was supported by the Russian Science Foundation, project 18-71-10097.

\section{References}

[1] M. Akian, A. Sulem, and M.I. Taksar. Dynamic optimization of long-term growth rate for a mixed portfolio with transactions costs, Mathematical Finance 11 (2001) 153-188.

[2] C. Albanese and S. Tompaidis. Small transaction cost asymptotics and dynamic hedging, European Journal of Operational Research 185 (2008) 1404-1414.

[3] P.H. Algoet and T.M. Cover. Asymptotic optimality and asymptotic equipartition properties of log-optimum investment, Annals of Probability 16 (1988) 876-898.

[4] S.V. Anoulova, I.V. Evstigneev, and V.M. Gundlach. Turnpike theorems for positive multivalued stochastic operators, Advances in Mathematical Economics 2 (2000) 1-20.

[5] W. Bahsoun, I.V. Evstigneev, and M.I. Taksar. Rapid paths in von Neumann-Gale dynamical systems, Stochastics 80 (2008) 129-142.

[6] W. Bahsoun, I.V. Evstigneev, and M.I. Taksar. Growth-optimal investments and numeraire portfolios under transaction costs, Handbook of the Fundamentals of Financial Decision Making, 2013, World Scientific, Singapore, pp. 789-808.

[7] B. Bouchard-Denize and N. Touzi. Explicit solution of the multivariate super-replication problem under transaction costs, Annals of Applied Probability 10 (2000) 685-708.

[8] L. Breiman. Optimal gambling systems for favorable games, Proceedings of the 4 th Berkeley Symposium on Mathematical Statistics and Probability, Vol. I, 1961, University of California Press, Berkeley, pp. 65-78.

[9] A. Cadenillas. Consumption-investment problems with transaction costs: Survey and open problems, Mathematical Methods of Operations Research 51 (2000) 43-68.

[10] A. Cadenillas and S. Pliska. Optimal trading of a security when there are taxes and transaction costs, Finance and Stochastics 3 (1999) 137-165.

[11] C. Castaing and M. Valadier. Convex Analysis and Measurable Multifunctions, Lecture Notes in Mathematics, no. 580, Springer-Verlag, Berlin-Heidelberg-New York, 1977.

[12] P. Chalasani and S. Jha. Randomized stopping times and American option pricing with transaction costs, Mathematical Finance 11 (2001) 33-77.

[13] S.A. Clark. Competitive prices for a stochastic input-output model with infinite time horizon, Economic Theory 35 (2008) 1-17.

[14] J. Cvitanić and I. Karatzas. Hedging and portfolio optimization under transaction costs: A martingale approach, Mathematical Finance 6 (1996) 133-165.

[15] S. Dasgupta and T. Mitra. Infinite-horizon competitive programs are optimal, Journal of Economics 69 (1999) 217-223.

[16] F. Delbaen, Yu.M. Kabanov, and E. Valkeila. Hedging under transaction costs in currency markets: a discrete-time model, Mathematical Finance 12 (2002) 45-61.

[17] M.A.H. Dempster, I.V. Evstigneev, and M.I. Taksar. Asset pricing and hedging in financial 
markets with transaction costs: An approach based on the von Neumann-Gale model, Annals of Finance 2 (2006) 327-355.

[18] E. Denis and Yu.M. Kabanov. Mean square error for the Leland-Lott hedging strategy: convex pay-offs, Finance and Stochastics 14 (2010) 625-667.

[19] D. De Vallière, Yu.M. Kabanov, and Ch. Stricker. No-arbitrage properties for financial markets with transaction costs and incomplete information, Finance and Stochastics 11 (2007) 237-251.

[20] E.B. Dynkin. Some probability models for a developing economy, Soviet Mathematics Doklady 12 (1971) 1422-1425.

[21] E.B. Dynkin. Stochastic concave dynamic programming, USSR Mathematics Sbornik 16 (1972) 501-515.

[22] E.B. Dynkin and A.A. Yushkevich. Controlled Markov Processes and their Applications, Springer, N.Y.4 1979.

[23] I.V. Evstigneev and K.R. Schenk-Hoppé. Pure and randomized equilibria in the stochastic von Neumann-Gale model, Journal of Mathematical Economics 43 (2007) 871-887.

[24] I.V. Evstigneev and K.R. Schenk-Hoppé. Stochastic equilibria in von Neumann-Gale dynamical systems, Transactions of the American Mathematical Society 360 (2008) 33453364.

[25] I.V. Evstigneev and M.V. Zhitlukhin. Controlled random fields, von Neumann-Gale dynamics and multimarket hedging with risk, Stochastics 85 (2013) 652-666.

[26] D. Gale. A closed linear model of production, in: H.W. Kuhn et al. (Eds.), Linear Inequalities and Related Systems, 1956, Princeton University Press, Princeton, pp. 285-303.

[27] D. Gale. On optimal development in a multi-sector economy, Review of Economic Studies 34 (1967) 1-18.

[28] P. Guasoni, E. Lépinette, and M. Rásonyi. The fundamental theorem of asset pricing under transaction costs, Finance and Stochastics 16 (2012) 741-777.

[29] P. Guasoni, M. Rásonyi, and W. Schachermayer. Consistent price systems and face-lifting pricing under transaction costs, Annals of Applied Probability 18 (2008) 491-520.

[30] N.H. Hakansson and W.T. Ziemba. Capital growth theory, in: R. Jarrow, A.V. Maksimovic, and W.T. Ziemba (Eds.), Handbooks in Operations Research and Management Science, Vol. 9, Finance, Chapter 3, 1995, Elsevier, Amsterdam, pp. 65-86.

[31] D.B. Hausch and W.T. Ziemba. Transactions costs, market inefficiencies and entries in a racetrack betting model, Management Science 31 (1985) 381-394.

[32] K. Janeček and S. Shreve. Asymptotic analysis for optimal investment and consumption with transaction costs, Finance and Stochastics 8 (2004) 181-206.

[33] D.G. Luenberger. Optimization by Vector Space Methods, Wiley, N. Y., 1997.

[34] G. Iyengar and T.M. Cover. Growth optimal investment in horse race markets with costs, IEEE Transactions on Information Theory 46 (2000) 2675-2683.

[35] G. Iyengar. Universal investment in markets with transaction costs, Mathematical Finance 15 (2005) 359-371.

[36] S. Jacka and A. Berkaoui. On the density of properly maximal claims in financial markets with transaction costs, Annals of Applied Probability 17 (2007) 716-740.

[37] S. Jacka, A. Berkaoui, and J. Warren. No arbitrage and closure results for trading cones with transaction costs, Finance and Stochastics 12 (2008) 583-600.

[38] E. Jouini and H. Kallal. Martingales and arbitrage in securities markets with transaction costs, Journal of Economic Theory 66 (1995) 178-197.

[39] Yu.M. Kabanov. Hedging and liquidation under transaction costs in currency markets, Finance and Stochastics 3 (1999) 237-248.

[40] Yu.M. Kabanov, M. Rásonyi, and Ch. Stricker. No-arbitrage criteria for financial markets with efficient friction, Finance and Stochastics 6 (2002) 371-382.

[41] Yu.M. Kabanov and M. Safarian. On Leland's strategy of option pricing with transaction costs, Finance and Stochastics 1 (1997) 239-250.

${ }^{4}$ Chapter 9 of this monograph presents the main results of the papers [20] and [21]. 
[42] Yu.M. Kabanov and M. Safarian. Markets with Transaction Costs, Springer-Verlag, Berlin Heidelberg, 2009.

[43] J.L. Kelly. A new interpretation of information rate, Bell System Technical Journal 35 (1956) 917-926.

[44] Yu. Kifer. Hedging of game options in discrete markets with transaction costs, Stochastics 85 (2013) 667-681.

[45] P. Koehl, H. Pham, and N. Touzi. Hedging in discrete time under transaction costs and continuous-time limit, Journal of Applied Probability 36 (1999) 163-178.

[46] P. Koehl, H. Pham, and N. Touzi. On super-replication in discrete time under transaction costs, Teoriya Veroyatnostei i ee Primeneniya 45 (2000) 783-788. English translation in Theory of Probability and Its Applications 45 (2002) 667-673.

[47] H. Latané. Criteria for choice among risky ventures, Journal of Political Economy 67 (1959) 144-155.

[48] H. Leland. Option pricing and replication with transactions costs, Journal of Finance $\mathbf{4 0}$ (1985) 1283-1301.

[49] H. Leland. Comments on "Hedging errors with Leland's option model in the presence of transactions costs", Finance Research Letters 4 (2007) 200-202.

[50] E. Lépinette. Modified Leland's strategy for a constant transaction costs rate, Mathematical Finance 4 (2012) 741-752.

[51] H. Liu and M. Loewenstein. Optimal portfolio selection with transaction costs and finite horizons, Review of Financial Studies 15 (2002) 805-835.

[52] J.B. Long. The numeraire portfolio, Journal of Financial Economics 26 (1990) 29-69.

[53] L.C. MacLean, W.T. Ziemba, and G. Blazenko. Growth versus security in dynamic investment analysis, Management Science 38 (1992) 1562-1585.

[54] L.C. MacLean, R. Sanegre, Y. Zhao, and W.T. Ziemba. Capital growth with security, Journal of Economic Dynamics and Control 28 (2004) 937-954.

[55] L.C. MacLean, W.T. Ziemba, and Y. Li. Time to wealth goals in capital accumulation, Quantitative Finance 5 (2005) 343-355.

[56] L.C. MacLean, E.O. Thorp, and W.T. Ziemba (Eds.). The Kelly Capital Growth Investment Criterion: Theory and Practice, World Scientific, Singapore, 2011.

[57] M.J.P. Magill and G.M. Constantinides. Portfolio selection with transaction costs, Journal of Economic Theory 13 (1976) 245-263.

[58] E. Malinvaud. Capital accumulation and efficient allocation of resources, Econometrica 21 (1953) 233-268.

[59] R.C. Merton. Optimum consumption and portfolio rules in a continuous time model, Journal of Economic Theory 3 (1971) 373-413.

[60] B. Peleg. On competitive prices for optimal consumption plans, SIAM Journal of Applied Mathematics 26 (1974) 239-253.

[61] H. Pham and N. Touzi. The fundamental theorem of asset pricing with cone constraints, Journal of Mathematical Economics 31 (1999) 265-279.

[62] E. Platen and D. Heath. A Benchmark Approach to Quantitative Finance, Springer, Heidelberg Dordrecht London New York, 2006.

[63] R. Radner. Efficiency prices for infinite horizon production programmes, Review of Economic Studies 34 (1967) 51-66.

[64] R. Radner. Balanced stochastic growth at the maximum rate, in: Contributions to the von Neumann Growth Model (Conference Proceedings), Institute for Advanced Studies, Vienna, 1970, Zeitschrift für Nationalökonomie, Suppl. No. 1, pp. 39-53.

[65] R. Radner. Optimal steady-state behavior of an economy with stochastic production and resources, in: R.H. Day and S.M. Robinson (Eds.), Mathematical Topics in Economic Theory and Computation, 1972, SIAM, Philadelphia, pp. 99-112.

[66] R.T. Rockafellar. Monotone Processes of Convex and Concave Type, Memoirs of the American Mathematical Society, Volume 77, American Mathematical Society, Providence, RI, 1967.

[67] A. Roux. Pricing and hedging game options in currency models with proportional transac- 
tion costs, International Journal of Theoretical and Applied Finance 19 (2016) 1650043.

[68] A. Roux and T. Zastawniak. American options under proportional transaction costs: Pricing, hedging and stopping algorithms for long and short positions, Acta Applicandae Mathematicae 106 (2009) 199-228.

[69] A. Roux and T. Zastawniak. American options with gradual exercise under proportional transaction costs, International Journal of Theoretical and Applied Finance 17 (2014) 1450052.

[70] A. Roux and T. Zastawniak. American and Bermudan options in currency markets with proportional transaction costs, Acta Applicandae Mathematicae 141 (2016) 187-225.

[71] A. Roux and T. Zastawniak. Game options with gradual exercise and cancellation under proportional transaction costs, Stochastics 90 (2018) 1190-1220.

[72] W. Schachermayer. The fundamental theorem of asset pricing under proportional transaction costs in finite discrete time, Mathematical Finance 14 (2004) 19-48.

[73] M.I. Taksar, M.J. Klass, and D. Assaf. Diffusion model for optimal portfolio selection in the presence of brokerage fees, Mathematics of Operations Research 13 (1988) 277-294.

[74] E.O. Thorp. Portfolio choice and the Kelly criterion, Proceedings of the Business and Economics Section of the American Statistical Association (1971) 215-224.

[75] Y. Zhao and W.T. Ziemba. Hedging errors with Leland's option model in the presence of transaction costs, Finance Research Letters 4 (2007) 49-58.

[76] Y. Zhao and W.T. Ziemba. Comments on and corrigendum to "Hedging errors with Leland's option model in the presence of transaction costs", Finance Research Letters 4 (2007) 196-199.

[77] W.T. Ziemba and R.G. Vickson (Eds.). Stochastic Optimization Models in Finance (2 ed.), World Scientific, Singapore, 2006.

[78] W.T. Ziemba and R.G. Vickson. Models of optimal capital accumulation and portfolio selection and the capital growth criterion, in: L.C. MacLean, E.O. Thorp, and W.T. Ziemba (Eds.), The Kelly Capital Growth Investment Criterion: Theory and Practice, 2011, World Scientific, Singapore, pp. 473-485.

[79] J. von Neumann. Über ein ökonomisches Gleichungssystem und eine Verallgemeinerung des Brouwerschen Fixpunktsatzes, in: Ergebnisse eines Mathematischen Kolloquiums 8 (1937) pp. 1935-1936 (Franz-Deuticke, Leipzig and Wien), pp. 73-83. [Translated: A model of general economic equilibrium, Review of Economic Studies 13 (1945-1946) 1-9.] 